\title{
A Theoretical Study of Substituent effects on Allylic Ion and Ion Pair $\mathrm{S}_{\mathrm{N}} 2$ Reactions
}

\author{
A. Streitwieser, ${ }^{*}$ E. G. Jayasree, S. S.-H. Leung and G. S.-C. Choy \\ Contribution from Department of Chemistry, University of California-Berkeley, Berkeley, \\ California 94720-1460 \\ astreit@,berkeley.edu \\ Supporting Information
}

\section{Contents}

$\begin{array}{ll}\text { Figure S1. Ion pair SN2 barriers vs } \sigma^{+} \text {constants. } & \text { p. S2 }\end{array}$

Table S1. Optimized coordinates and energies, HF 6-31+G* p. S3

Table S2. Energy barriers of ion-pair $\mathrm{S}_{\mathrm{N}} 2$ reactions of MX with allyl X. p. S35

$\begin{array}{ll}\text { Table S3. Reduced natural charges } & \text { p. S37 }\end{array}$

Allyl SN2 SI v2a.doc - S1- 


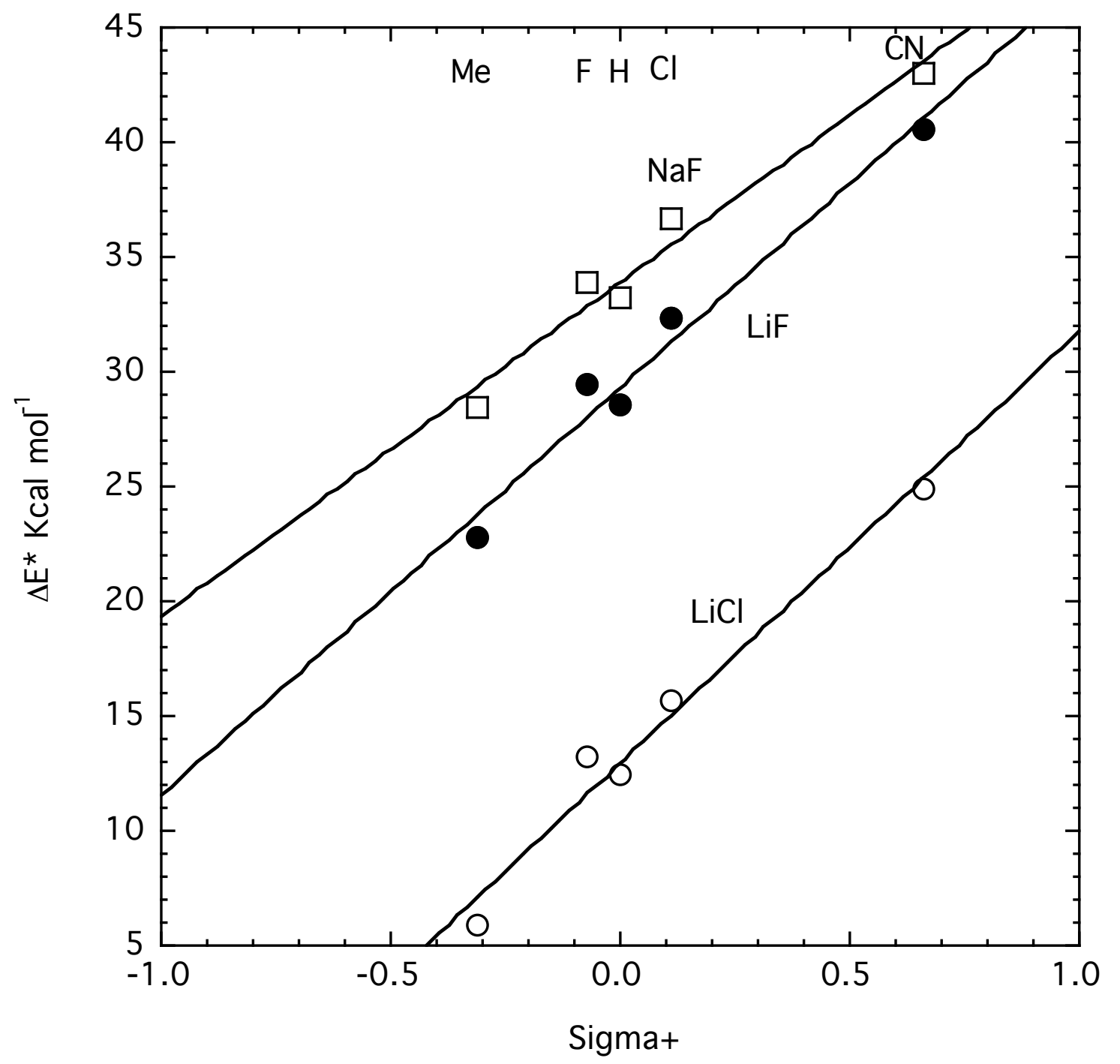

Figure S1. Ion pair SN2 barriers vs $\sigma^{+}$constants. Lines are: NaF, $33.90 \pm 0.53+(14.58 \pm$ 1.61) $\sigma^{+}, \mathrm{R}^{2}=0.965 ; \mathrm{LiF}, 29.31 \pm 0.59+(17.77 \pm 1.77) \sigma^{+}, \mathrm{R}^{2}=0.971 ; \mathrm{LiCl}, 12.96 \pm$ $0.59+(18.80 \pm 1.78) \sigma^{+}, R^{2}=0.974$. Similar plots are found vs $\sigma: \mathrm{NaF}, 32.43 \pm 0.46+$ $(16.74 \pm 1.42) \sigma, \mathrm{R}^{2}=0.979 ; \mathrm{LiF}, 27.52 \pm 0.54+(20.35 \pm 1.69) \sigma, \mathrm{R}^{2}=0.980 ; \mathrm{LiCl}$, $11.08 \pm 0.68+(21.42 \pm 2.09) \sigma, R^{2}=0.972$.

Table S1. Optimized coordinates of the ionic, ion pair transition structures and reactants, their Hartree-Fock energies and zero point energies (au) at $6-31+\mathrm{G}^{*}$. All transition structures have one imaginay frequency. All normal structures have all frequencies real. 


\section{Ionic transition structures}

allyl chloride + chloride ion

-1035.501003 au

$0.0759537 \mathrm{au}$

$\begin{array}{lccc}6 & .000000 & .273235 & .000000 \\ 6 & -.506264 & -1.098845 & .000000 \\ 6 & .293502 & -2.150973 & .000000 \\ 1 & -1.574137 & -1.212617 & .000000 \\ 1 & -.661330 & 1.101714 & .000000 \\ 1 & 1.045952 & .464756 & .000000 \\ 1 & -.099108 & -3.151945 & .000000 \\ 1 & 1.363619 & -2.045659 & .000000 \\ 17 & .035341 & .667743 & 2.465192 \\ 17 & .035341 & .667743 & -2.465192\end{array}$

(trans)methyl allyl chloride+chloride ion

$-1074.541672$

0.105911 au

$\begin{array}{llcc}6 & 0.000000 & 0.715674 & 0.000000 \\ 6 & -0.492987 & -0.651154 & 0.000000 \\ 6 & 0.326392 & -1.691054 & 0.000000 \\ 1 & -1.559944 & -0.781004 & 0.000000 \\ 1 & -0.666409 & 1.541056 & 0.000000 \\ 1 & 1.043946 & 0.918527 & 0.000000 \\ 1 & 1.389368 & -1.512564 & 0.000000 \\ 17 & 0.060780 & 1.142347 & 2.493760 \\ 17 & 0.060780 & 1.142347 & -2.493760 \\ 6 & -0.107252 & -3.125087 & 0.000000 \\ 1 & -1.187855 & -3.213767 & 0.000000 \\ 1 & 0.278729 & -3.641161 & -0.876030 \\ 1 & 0.278729 & -3.641161 & 0.876030\end{array}$

(trans) $\mathrm{SiH} 3$ allyl chloride + chloride ion $-1325.5844182$ $0.0925068 \mathrm{au}$

$\begin{array}{lrrr}\mathrm{H} & -2.6445727 & 0.0004911 & 0.6964600 \\ \mathrm{C} & -1.8672815 & -0.0015566 & -0.0243553 \\ \mathrm{H} & -2.1301410 & -0.0051820 & -1.0546307 \\ \mathrm{Cl} & -2.2033914 & 2.4417101 & -0.0183555 \\ \mathrm{Cl} & -2.1985393 & -2.4415991 & -0.0063862 \\ \mathrm{C} & -0.4556213 & 0.0028834 & 0.3853738 \\ \mathrm{C} & 0.5536697 & -0.0005268 & -0.4771778 \\ \mathrm{H} & 0.3141723 & -0.0062555 & -1.5303239 \\ \mathrm{H} & -0.2891801 & 0.0082261 & 1.4476465 \\ \mathrm{Si} & 2.3447975 & 0.0005134 & 0.0384116 \\ \mathrm{H} & 2.4537369 & 0.0034110 & 1.5125009 \\ \mathrm{H} & 3.0621434 & 1.1849996 & -0.4869705 \\ \mathrm{H} & 3.0602075 & -1.1871148 & -0.4821928\end{array}$


(trans) $\mathrm{OH}$ allylchloride + chloride ion

$-1110.36524771$

$0.081739 \mathrm{au}$

$\begin{array}{lrcc}6 & 0.000000 & 0.632719 & 0.000000 \\ 6 & -0.565273 & -0.679868 & 0.000000 \\ 6 & 0.227872 & -1.746987 & 0.000000 \\ 1 & -1.636197 & -0.776743 & 0.000000 \\ 1 & -0.615441 & 1.497564 & 0.000000 \\ 1 & 1.053616 & 0.780586 & 0.000000 \\ 1 & 1.296972 & -1.667507 & 0.000000 \\ 17 & 0.130164 & 1.122524 & 2.543816 \\ 17 & 0.130164 & 1.122524 & -2.543816 \\ 8 & -0.172511 & -3.020110 & 0.000000 \\ 1 & -1.120046 & -3.073999 & 0.000000\end{array}$

(trans)F allyl chloride+chloride ion

$-1134.359258$

0.068286 au

$\begin{array}{lrrr}6 & 0.000000 & 0.676356 & 0.000000 \\ 6 & -0.534337 & -0.673723 & 0.000000 \\ 6 & 0.277931 & -1.705934 & 0.000000 \\ 1 & -1.598026 & -0.809759 & 0.000000 \\ 1 & -0.647371 & 1.515929 & 0.000000 \\ 1 & 1.047990 & 0.853156 & 0.000000 \\ 1 & 1.347982 & -1.663802 & 0.000000 \\ 17 & 0.087497 & 1.086205 & 2.471134 \\ 17 & 0.087497 & 1.086205 & -2.471134 \\ 9 & -0.176337 & -2.956297 & 0.000000\end{array}$

(trans) $\mathrm{CN}$ allyl chloride + chloride ion

$-1127.248479$

$0.075529 \mathrm{au}$

$\begin{array}{lrcc}6 & 0.785810 & -0.731264 & 0.000000 \\ 6 & 0.000000 & 0.512804 & 0.000000 \\ 6 & -1.324362 & 0.521667 & 0.000000 \\ 1 & 0.558755 & 1.428699 & 0.000000 \\ 1 & 1.844622 & -0.697596 & 0.000000 \\ 1 & 0.301458 & -1.677105 & 0.000000 \\ 1 & -1.898753 & -0.385188 & 0.000000 \\ 17 & 0.994141 & -0.878625 & 2.400104 \\ 17 & 0.994141 & -0.878625 & -2.400104 \\ 6 & -2.078259 & 1.746889 & 0.000000 \\ 7 & -2.700857 & 2.700552 & 0.000000\end{array}$

(trans) Cl allyl chloride + chloride ion

$-1494.405167$

$0.066723 \mathrm{au}$

$\begin{array}{llll}6 & 0.962741 & -0.579921 & 0.000000\end{array}$

$\begin{array}{lllll}6 & 0.000000 & 0.521078 & 0.000000\end{array}$

Allyl SN2 SI v2a.doc

- S4- 


$\begin{array}{crrr}6 & -1.294488 & 0.287486 & 0.000000 \\ 1 & 0.390380 & 1.519891 & 0.000000 \\ 1 & 2.004339 & -0.384678 & 0.000000 \\ 1 & 0.636996 & -1.591446 & 0.000000 \\ 1 & -1.725159 & -0.692544 & 0.000000 \\ 17 & 1.260737 & -0.783760 & 2.443702 \\ 17 & 1.260737 & -0.783760 & -2.443702 \\ 17 & -2.481243 & 1.554397 & 0.000000\end{array}$

(cis)methyl allyl chloride + chloride ion $-1074.539823$

0.106114 au

$\begin{array}{cccc}6 & 0.000000 & 0.610320 & 0.000000 \\ 6 & -1.036675 & -0.412786 & 0.000000 \\ 6 & -0.817320 & -1.721009 & 0.000000 \\ 1 & -2.046498 & -0.047148 & 0.000000 \\ 1 & -0.264432 & 1.637855 & 0.000000 \\ 1 & 1.034057 & 0.372649 & 0.000000 \\ 1 & -1.684928 & -2.362250 & 0.000000 \\ 17 & 0.252638 & 0.943023 & 2.483025 \\ 17 & 0.252638 & 0.943023 & -2.483025 \\ 6 & 0.500068 & -2.435646 & 0.000000 \\ 1 & 1.345123 & -1.763241 & 0.000000 \\ 1 & 0.575278 & -3.072954 & 0.877686 \\ 1 & 0.575278 & -3.072954 & -0.877686\end{array}$

(cis) $\mathrm{SiH} 3$ allyl chloride + chloride ion $-1325.581827$

$0.092824 \mathrm{au}$

$\begin{array}{llll}6 & 0.583022 & -1.160418 & 1.041706 \\ 1 & 0.745948 & -1.683574 & 1.973840 \\ 6 & -0.193205 & -0.084880 & 1.113936 \\ 1 & -0.614754 & 0.216045 & 2.056280 \\ 6 & -0.563263 & 0.782242 & -0.015082 \\ 1 & -0.377343 & 0.502048 & -1.022312 \\ 1 & -1.060096 & 1.702247 & 0.161896 \\ 17 & -2.855535 & -0.107299 & -0.276217 \\ 17 & 1.476467 & 2.146647 & -0.151960 \\ 14 & 1.471525 & -1.876617 & -0.445027 \\ 1 & 1.188291 & -3.331423 & -0.499479 \\ 1 & 1.067421 & -1.279177 & -1.725842 \\ 1 & 2.934008 & -1.744108 & -0.278362\end{array}$

(cis) $\mathrm{OH}$ allyl chloride + chloride ion $-1110.356213$

$0.081223 \mathrm{au}$

$\begin{array}{rrrr}6 & 0.621724 & 1.797780 & 0.000000 \\ 1 & 0.508406 & 2.868050 & 0.000000 \\ 6 & -0.441194 & 1.005669 & 0.000000 \\ 1 & -1.414021 & 1.455241 & 0.000000\end{array}$




$\begin{array}{llll}6 & -0.350979 & -0.432725 & 0.000000 \\ 1 & 0.593331 & -0.921649 & 0.000000 \\ 1 & -1.226501 & -1.031502 & 0.000000 \\ 17 & -0.441194 & -0.864615 & 2.520213 \\ 17 & -0.441194 & -0.864615 & -2.520213 \\ 8 & 1.882116 & 1.343630 & 0.000000 \\ 1 & 2.505157 & 2.053378 & 0.000000\end{array}$

(cis) $\mathrm{F}$ allyl chloride + chloride ion

$-1134.356490$

0.068675 au

$\begin{array}{llll}6 & 0.609820 & 1.785035 & 0.000000 \\ 1 & 0.552235 & 2.854683 & 0.000000 \\ 6 & -0.426158 & 0.976271 & 0.000000 \\ 1 & -1.404603 & 1.413866 & 0.000000 \\ 6 & -0.327184 & -0.476997 & 0.000000 \\ 1 & 0.619873 & -0.959108 & 0.000000 \\ 1 & -1.202330 & -1.074197 & 0.000000 \\ 9 & 1.865037 & 1.355294 & 0.000000 \\ 17 & -0.426158 & -0.827610 & 2.459950 \\ 17 & -0.426158 & -0.827610 & -2.459950\end{array}$

(cis) $\mathrm{CN}$ allyl chloride + chloride ion

$-1127.243875$

0.075859 au

$\begin{array}{lccc}6 & 0.324039 & 1.699273 & 0.000000 \\ 1 & 0.110163 & 2.751148 & 0.000000 \\ 6 & -0.664379 & 0.815782 & 0.000000 \\ 1 & -1.673907 & 1.177785 & 0.000000 \\ 6 & -0.518581 & -0.648543 & 0.000000 \\ 1 & 0.443808 & -1.102817 & 0.000000 \\ 1 & -1.378490 & -1.265989 & 0.000000 \\ 17 & -0.664379 & -0.854535 & 2.398908 \\ 17 & -0.664379 & -0.854535 & -2.398908 \\ 6 & 1.723327 & 1.363619 & 0.000000 \\ 7 & 2.842984 & 1.159041 & 0.000000\end{array}$

(cis) Cl allyl chloride + chloride ion $-1494.401913$

0.067155 au

$\begin{array}{lccc}6 & 0.275642 & 1.651558 & 0.000000 \\ 1 & 0.105971 & 2.708551 & 0.000000 \\ 6 & -0.716084 & 0.783987 & 0.000000 \\ 1 & -1.714090 & 1.179237 & 0.000000 \\ 6 & -0.598116 & -0.674987 & 0.000000 \\ 1 & 0.352211 & -1.150535 & 0.000000 \\ 1 & -1.469812 & -1.276268 & 0.000000 \\ 17 & -0.716084 & -0.977466 & 2.434935 \\ 17 & -0.716084 & -0.977466 & -2.434935 \\ 17 & 1.959055 & 1.247618 & 0.000000\end{array}$




\section{Ion Pair Transition structures}

$$
\begin{aligned}
& \text { allyl fluoride + LIF } \\
& -322.828743 \\
& 0.0795797 \mathrm{au} \\
& \begin{array}{llll}
6 & -.215278 & .411751 & .000000 \\
6 & -.215278 & 1.825437 & .000000 \\
6 & .967385 & 2.463334 & .000000 \\
3 & -.231824 & -2.397416 & .000000 \\
9 & -.215278 & -1.585614 & 1.484749 \\
9 & -.215278 & -1.585614 & -1.484749 \\
1 & .689872 & -.147100 & .000000 \\
1 & -1.120428 & -.147100 & .000000 \\
1 & -1.144142 & 2.364036 & .000000 \\
1 & 1.897133 & 1.923621 & .000000 \\
1 & 1.027071 & 3.536721 & .000000
\end{array} \\
& \text { 2-F allyl fluoride }+\mathrm{LiF} \\
& -421.673891 \mathrm{au} \\
& 0.0712173 \mathrm{au}
\end{aligned}
$$

$\begin{array}{llll}6 & .000000 & .071025 & .000000 \\ 6 & 1.408799 & .142841 & .000000 \\ 6 & 2.159096 & -.960391 & .000000 \\ 3 & -2.770823 & -.282530 & .000000 \\ 9 & -1.923947 & -.223242 & 1.469198 \\ 9 & -1.923947 & -.223242 & -1.469198 \\ 1 & -.483134 & -.873581 & .000000 \\ 1 & -.603940 & .948009 & .000000 \\ 9 & 1.966932 & 1.344938 & .000000 \\ 1 & 1.688875 & -1.923948 & .000000 \\ 1 & 3.231963 & -.909836 & .000000\end{array}$

2-Me allyl fluoride $+\mathrm{LiF}$ $-361.868201$

0.1093294 au

$\begin{array}{lccc}6 & .000000 & .012939 & .000000 \\ 6 & -.793233 & 1.188972 & .000000 \\ 6 & -.098944 & 2.341986 & .000000 \\ 3 & 1.633771 & -2.276447 & .000000 \\ 9 & 1.182529 & -1.610357 & 1.488133 \\ 9 & 1.182529 & -1.610357 & -1.488133 \\ 1 & 1.062088 & .060935 & .000000 \\ 1 & -.428778 & -.961990 & .000000 \\ 6 & -2.300687 & 1.098588 & .000000 \\ 1 & .975463 & 2.344809 & .000000 \\ 1 & -.585209 & 3.301466 & .000000 \\ 1 & -2.749851 & 2.083920 & .000000 \\ 1 & -2.651679 & .565863 & -.876888 \\ 1 & -2.651679 & .565863 & .876888\end{array}$




$\begin{array}{lccl}\text { allyl chloride }+\mathrm{LiCl} \\ -1042.967082 \mathrm{au} \\ 0.0779054 \mathrm{au} \\ 6 & -.157908 & .996425 & .000000 \\ 6 & -.157908 & 2.403734 & .000000 \\ 6 & 1.028150 & 3.042021 & .000000 \\ 3 & -.182839 & -2.247053 & .000000 \\ 17 & -.157908 & -1.245557 & 1.974735 \\ 17 & -.157908 & -1.245557 & -1.974735 \\ 1 & .751789 & .439243 & .000000 \\ 1 & -1.067606 & .439243 & .000000 \\ 1 & -1.086623 & 2.941969 & .000000 \\ 1 & 1.957214 & 2.501278 & .000000 \\ 1 & 1.088621 & 4.115290 & .000000 \\ 5-\mathrm{F} \text { allyl chloride }+\mathrm{LiCl} & & \\ -1141.810803 \mathrm{au} & & \\ 0.069564 \text { au } & & \\ 5 & & & \\ 6 & 1.095252 & 2.661236 & 0.000000 \\ 1 & 2.020584 & 2.119390 & 0.000000 \\ 1 & 1.125959 & 3.734824 & 0.000000 \\ 6 & -0.065557 & 1.997977 & 0.000000 \\ 6 & -0.104352 & 0.592923 & 0.000000 \\ 17 & 0.067414 & -1.571459 & 1.958367 \\ 17 & 0.067414 & -1.571459 & -1.958367 \\ 3 & 0.067414 & -2.621580 & 0.000000 \\ 1 & -1.029716 & 0.061092 & 0.000000 \\ 1 & 0.805310 & 0.041974 & 0.000000 \\ 9 & -1.218725 & 2.647139 & 0.000000\end{array}$

2-Me allyl chloride + $\mathrm{LiCl}$

-1082.006962 au

0.107652 au

$\begin{array}{lrrr}6 & -2.536681 & -0.000118 & 1.344956 \\ 1 & -1.891441 & -0.000282 & 2.204087 \\ 1 & -3.595214 & 0.000098 & 1.535141 \\ 6 & -2.036018 & -0.000133 & 0.091893 \\ 6 & -0.626153 & -0.000069 & 0.016534 \\ 17 & 1.631272 & -1.979143 & -0.023011 \\ 17 & 1.631104 & 1.979231 & -0.022937 \\ 3 & 2.620244 & 0.000064 & -0.073144 \\ 1 & -0.108514 & 0.000279 & -0.917699 \\ 1 & -0.025146 & -0.000277 & 0.897170 \\ 6 & -2.875035 & 0.000033 & -1.164017 \\ 1 & -2.663354 & -0.877342 & -1.764766 \\ 1 & -2.663181 & 0.877439 & -1.764660 \\ 1 & -3.930945 & 0.000116 & -0.924922\end{array}$

allyl fluoride $+\mathrm{NaF}$

-477.196166 au 


\begin{tabular}{lccc}
$0.0783427 \mathrm{au}$ \\
\multicolumn{4}{l}{$l$} \\
6 & -.174448 & .712365 & .000000 \\
6 & -.174448 & 2.136288 & .000000 \\
6 & .998915 & 2.780498 & .000000 \\
11 & -.207971 & -2.264049 & .000000 \\
9 & -.174448 & -.998882 & 1.637964 \\
9 & -.174448 & -.998882 & -1.637964 \\
1 & .735209 & .169406 & .000000 \\
1 & -1.084105 & .169406 & .000000 \\
1 & -1.107194 & 2.668414 & .000000 \\
1 & 1.932779 & 2.248052 & .000000 \\
1 & 1.050942 & 3.854227 & .000000
\end{tabular}

2-F allyl fluoride $+\mathrm{NaF}$

-576.043213 au

$0.070069 \mathrm{au}$

$\begin{array}{lccc}6 & 1.069574 & 2.447210 & 0.000000 \\ 1 & 2.019746 & 1.951030 & 0.000000 \\ 1 & 1.048654 & 3.520803 & 0.000000 \\ 6 & -0.048587 & 1.730666 & 0.000000 \\ 6 & -0.034551 & 0.307439 & 0.000000 \\ 9 & 0.076059 & -1.326861 & 1.620969 \\ 9 & 0.076059 & -1.326861 & -1.620969 \\ 11 & 0.076059 & -2.631684 & 0.000000 \\ 1 & -0.944072 & -0.235583 & 0.000000 \\ 1 & 0.895301 & -0.194099 & 0.000000 \\ 9 & -1.238217 & 2.319775 & 0.000000\end{array}$

2-Me allyl fluoride $+\mathrm{NaF}$

-516.235485 au

$0.108088 \mathrm{au}$

$\begin{array}{lccc}6 & 2.283596 & -0.000768 & 1.325472 \\ 1 & 1.661477 & -0.001247 & 2.201381 \\ 1 & 3.346928 & -0.000925 & 1.487799 \\ 6 & 1.751538 & -0.000033 & 0.095099 \\ 6 & 0.323595 & -0.000028 & 0.040868 \\ 9 & -1.395577 & 1.643087 & -0.007122 \\ 9 & -1.395623 & -1.643092 & -0.008410 \\ 11 & -2.652200 & 0.000056 & -0.065891 \\ 1 & -0.192707 & 0.000390 & -0.885172 \\ 1 & -0.250007 & -0.000405 & 0.931425 \\ 6 & 2.552813 & 0.000672 & -1.183926 \\ 1 & 2.321817 & 0.878008 & -1.778524 \\ 1 & 2.322095 & -0.876199 & -1.779320 \\ 1 & 3.616146 & 0.000751 & -0.978068 \\ \text { trans)F allyl fluoride }+ \text { LiF } & \\ -421.681373 & \\ 0.072102 & \end{array}$

Allyl SN2 SI v2a.doc - S9- 


$\begin{array}{rrrr}6 & 0.458142 & 0.253046 & 0.000000 \\ 6 & 0.784676 & -1.104719 & 0.000000 \\ 6 & -0.260738 & -1.954278 & 0.000000 \\ 3 & -0.472945 & 2.934573 & 0.000000 \\ 9 & -0.260738 & 2.146292 & 1.480952 \\ 9 & -0.260738 & 2.146292 & -1.480952 \\ 1 & -0.551501 & 0.586230 & 0.000000 \\ 1 & 1.201061 & 1.016870 & 0.000000 \\ 1 & 1.794803 & -1.466982 & 0.000000 \\ 9 & -0.103694 & -3.231632 & 0.000000 \\ 1 & -1.291487 & -1.652699 & 0.000000\end{array}$

(cis) F allyl fluoride + LiF $-421.686882$

$0.0722489 \mathrm{au}$

$\begin{array}{lccc}6 & .000000 & .560736 & .000000 \\ 6 & 1.377286 & .835757 & .000000 \\ 6 & 2.274683 & -.167527 & .000000 \\ 3 & -2.746826 & -.013757 & .000000 \\ 9 & -1.950022 & .147391 & 1.484596 \\ 9 & -1.950022 & .147391 & -1.484596 \\ 1 & -.372576 & -.435110 & .000000 \\ 1 & -.720286 & 1.343000 & .000000 \\ 1 & 1.747200 & 1.842628 & .000000 \\ 9 & 1.937268 & -1.411937 & .000000 \\ 1 & 3.339313 & -.028644 & .000000\end{array}$

(trans) Cl allyl fluoride $+\mathrm{LiF}$ -781.727594 au $0.070287 \mathrm{au}$

$\begin{array}{llll}6 & -0.250058 & -1.429627 & 0.000000 \\ 6 & 0.772813 & -0.551902 & 0.000000 \\ 6 & 0.427156 & 0.809144 & 0.000000 \\ 9 & -0.250058 & 2.703149 & 1.482092 \\ 9 & -0.250058 & 2.703149 & -1.482092 \\ 3 & -0.465132 & 3.489594 & 0.000000 \\ 1 & -0.587461 & 1.126255 & 0.000000 \\ 1 & 1.795587 & -0.876798 & 0.000000 \\ 1 & 1.162551 & 1.579686 & 0.000000 \\ 1 & -1.273206 & -1.104260 & 0.000000 \\ 17 & -0.052970 & -3.106826 & 0.000000\end{array}$

(cis) $\mathrm{Cl}$ allyl fluoride $+\mathrm{LiF}$ $-781.721751 \mathrm{au}$ 0.070368 au

\begin{tabular}{|c|c|c|c|}
\hline 6 & 1.031428 & -1.750766 & 0.000000 \\
\hline 6 & 1.492791 & -0.483173 & 0.000000 \\
\hline 6 & 0.640217 & 0.638449 & 0.00000 \\
\hline 9 & -0.589758 & 2.207012 & 1.485286 \\
\hline 9 & -0.589758 & 2.207012 & -1.48528 \\
\hline
\end{tabular}




$\begin{array}{llcc}3 & -1.078283 & 2.855769 & 0.000000 \\ 1 & -0.418310 & 0.545494 & 0.000000 \\ 1 & 2.557845 & -0.344588 & 0.000000 \\ 1 & 1.716472 & -2.577259 & 0.000000 \\ 17 & -0.589758 & -2.233570 & 0.000000 \\ 1 & 1.033744 & 1.626465 & 0.000000\end{array}$

(trans) $\mathrm{CN}$ allyl fluoride $+\mathrm{LiF}$

-414.549564 au

0.078738 au

$\begin{array}{rrrr}6 & -0.272637 & -1.572307 & 0.000000 \\ 6 & 0.719600 & -0.670262 & 0.000000 \\ 6 & 0.354263 & 0.707223 & 0.000000 \\ 9 & -0.272637 & 2.534923 & 1.469318 \\ 9 & -0.272637 & 2.534923 & -1.469318 \\ 3 & -0.504060 & 3.356955 & 0.000000 \\ 1 & -0.666438 & 1.000126 & 0.000000 \\ 1 & 1.751817 & -0.964687 & 0.000000 \\ 1 & 1.084441 & 1.480378 & 0.000000 \\ 1 & -1.304208 & -1.268900 & 0.000000 \\ 6 & -0.034972 & -2.990582 & 0.000000 \\ 7 & 0.136644 & -4.112976 & 0.000000\end{array}$

(cis) $\mathrm{CN}$ allyl fluoride $+\mathrm{LiF}$

-414.544885 au

0.078849 au

$\begin{array}{lrrr}6 & 0.979963 & -1.916375 & 0.000000 \\ 6 & 1.493264 & -0.677644 & 0.000000 \\ 6 & 0.656822 & 0.482313 & 0.000000 \\ 9 & -0.425129 & 2.051441 & 1.470456 \\ 9 & -0.425129 & 2.051441 & -1.470456 \\ 3 & -0.930597 & 2.742480 & 0.000000 \\ 1 & -0.403296 & 0.403256 & 0.000000 \\ 1 & 2.559538 & -0.555100 & 0.000000 \\ 1 & 1.079501 & 1.455954 & 0.000000 \\ 1 & 1.628839 & -2.773882 & 0.000000 \\ 6 & -0.425129 & -2.227193 & 0.000000 \\ 7 & -1.521426 & -2.521459 & 0.000000\end{array}$

(trans)Me allyl fluoride + LiF

$-361.878012 \mathrm{au}$

0.109383 au

$\begin{array}{rrrr}6 & 0.435754 & 0.304879 & 0.000000 \\ 6 & 0.763203 & -1.054540 & 0.000000 \\ 6 & -0.252684 & -1.952408 & 0.000000 \\ 3 & -0.455659 & 3.002934 & 0.000000 \\ 9 & -0.252684 & 2.247402 & 1.494976 \\ 9 & -0.252684 & 2.247402 & -1.494976 \\ 1 & -0.576212 & 0.632572 & 0.000000 \\ 1 & 1.175187 & 1.072729 & 0.000000\end{array}$




$\begin{array}{lrrr}1 & 1.789503 & -1.373429 & 0.000000 \\ 6 & -0.102925 & -3.430843 & 0.000000 \\ 1 & -1.260520 & -1.570978 & 0.000000 \\ 1 & 0.933266 & -3.739901 & 0.000000 \\ 1 & -0.603013 & -3.842778 & 0.872433 \\ 1 & -0.603013 & -3.842778 & -0.872433\end{array}$

(cis)Me allyl fluoride + LiF

$-361.874023 \mathrm{au}$

0.1093873 au

$\begin{array}{lccc}6 & .661140 & -.348476 & .000000 \\ 6 & .222661 & -1.675189 & .000000 \\ 6 & -1.102827 & -1.981103 & .000000 \\ 3 & 1.218699 & 2.460570 & .000000 \\ 9 & .990385 & 1.708385 & 1.492347 \\ 9 & .990385 & 1.708385 & -1.492347 \\ 1 & .000000 & .480481 & .000000 \\ 1 & 1.699513 & -.102122 & .000000 \\ 1 & .943584 & -2.471572 & .000000 \\ 6 & -2.276842 & -1.065197 & .000000 \\ 1 & -1.354147 & -3.029807 & .000000 \\ 1 & -2.024941 & -.017215 & .000000 \\ 1 & -2.885913 & -1.286307 & -.872304 \\ 1 & -2.885913 & -1.286307 & .872304\end{array}$

(trans) SH allyl fluoride $+\mathrm{LiF}$ -720.348859 au 0.079844 au

$\begin{array}{lrrr}6 & -0.233999 & -1.423440 & 0.000000 \\ 6 & 0.830352 & -0.566487 & 0.000000 \\ 6 & 0.542546 & 0.789846 & 0.000000 \\ 9 & -0.233999 & 2.745963 & 1.497451 \\ 9 & -0.233999 & 2.745963 & -1.497451 \\ 3 & -0.425526 & 3.494110 & 0.000000 \\ 1 & -0.457367 & 1.152555 & 0.000000 \\ 1 & 1.842014 & -0.928232 & 0.000000 \\ 1 & 1.305272 & 1.536492 & 0.000000 \\ 1 & -1.228428 & -1.014515 & 0.000000 \\ 16 & -0.088940 & -3.127860 & 0.000000 \\ 1 & -1.383293 & -3.409728 & 0.000000\end{array}$

(cis) SH allyl fluoride $+\mathrm{LiF}$

-720.341777 au

$0.079709 \mathrm{au}$

$\begin{array}{lrrr}6 & 1.027463 & -1.745109 & 0.000000 \\ 6 & 1.554959 & -0.480362 & 0.000000 \\ 6 & 0.754305 & 0.653441 & 0.000000 \\ 9 & -0.632558 & 2.215329 & 1.497080 \\ 9 & -0.632558 & 2.215329 & -1.497080 \\ 3 & -1.091315 & 2.839085 & 0.000000\end{array}$




$\begin{array}{llcc}1 & -0.309125 & 0.613403 & 0.000000 \\ 1 & 2.624557 & -0.385663 & 0.000000 \\ 1 & 1.172989 & 1.634026 & 0.000000 \\ 1 & 1.715958 & -2.571367 & 0.000000 \\ 16 & -0.632558 & -2.172980 & 0.000000 \\ 1 & -0.443831 & -3.483713 & 0.000000\end{array}$

(trans) $\mathrm{OH}$ allyl fluoride $+\mathrm{LiF}$ -397.685158 au 0.084936 au

\begin{tabular}{|c|c|c|c|}
\hline 6 & 1.643900 & -1.086032 & 0.000000 \\
\hline 6 & 1.344224 & 0.247161 & 0.000000 \\
\hline 6 & 0.000000 & 0.587063 & 0.000000 \\
\hline 9 & -2.098077 & 0.752860 & 1.497451 \\
\hline 9 & -2.098077 & 0.752860 & -1.497451 \\
\hline 3 & -2.853999 & 0.910934 & 0.000000 \\
\hline 1 & -0.766532 & -0.150373 & 0.000000 \\
\hline 1 & 2.115073 & 0.995567 & 0.000000 \\
\hline 1 & -0.333707 & 1.600902 & 0.000000 \\
\hline 1 & 0.838308 & -1.798164 & 0.000000 \\
\hline 8 & 2.976164 & -1.605621 & 0.000000 \\
\hline 1 & 2.736187 & -2.576399 & 0.000000 \\
\hline
\end{tabular}

(cis) $\mathrm{OH}$ allyl fluoride $+\mathrm{LiF}$ -397.700505 au 0.085739 au

\begin{tabular}{|c|c|c|c|}
\hline 6 & 0.679874 & -2.192614 & 0.000000 \\
\hline 6 & 1.315912 & -0.979108 & 0.000000 \\
\hline 6 & 0.588227 & 0.197053 & 0.000000 \\
\hline 9 & -0.607626 & 1.924663 & 1.508432 \\
\hline 9 & -0.607626 & 1.924663 & -1.508432 \\
\hline 3 & -1.009088 & 2.556877 & 0.000000 \\
\hline 1 & -0.476047 & 0.203781 & 0.000000 \\
\hline 1 & 2.388621 & -0.978418 & 0.000000 \\
\hline 1 & 1.064457 & 1.150271 & 0.000000 \\
\hline 1 & 1.254301 & -3.104395 & 0.000000 \\
\hline 8 & -0.607626 & -2.315254 & 0.000000 \\
\hline 1 & -0.909861 & -3.215759 & 0.000000 \\
\hline
\end{tabular}

(trans-anti)vinyl allyl fluoride $+\mathrm{LiF}$ -399.730514 au 0.115709 au

$\begin{array}{lrrr}6 & 0.115457 & -1.502392 & 0.000000 \\ 6 & 1.014063 & -0.473896 & 0.000000 \\ 6 & 0.517462 & 0.823942 & 0.000000 \\ 9 & -0.489842 & 2.673933 & 1.502727 \\ 9 & -0.489842 & 2.673933 & -1.502727 \\ 3 & -0.784940 & 3.374591 & 0.000000 \\ 1 & -0.528067 & 1.021933 & 0.000000 \\ 1 & 2.071975 & -0.662765 & 0.000000\end{array}$




$\begin{array}{lrrr}1 & 1.151282 & 1.682060 & 0.000000 \\ 1 & -0.933260 & -1.256599 & 0.000000 \\ 6 & 0.458043 & -2.904956 & 0.000000 \\ 1 & 1.499620 & -3.172107 & 0.000000 \\ 6 & -0.489842 & -3.841246 & 0.000000 \\ 1 & -1.535416 & -3.588519 & 0.000000 \\ 1 & -0.245255 & -4.887276 & 0.000000\end{array}$

(cis-anti)vinyl allyl fluoride + LiF $-399.726050 \mathrm{au}$

0.115666 au

$\begin{array}{lrrr}6 & 0.903426 & -1.733374 & 0.000000 \\ 6 & 1.522125 & -0.519352 & 0.000000 \\ 6 & 0.798568 & 0.679397 & 0.000000 \\ 9 & -0.519740 & 2.322318 & 1.502727 \\ 9 & -0.519740 & 2.322318 & -1.502727 \\ 3 & -0.933985 & 2.959817 & 0.000000 \\ 1 & 1.294307 & 1.620474 & 0.000000 \\ 1 & -0.258681 & 0.710902 & 0.000000 \\ 1 & 2.595515 & -0.484726 & 0.000000 \\ 1 & 1.537373 & -2.605013 & 0.000000 \\ 6 & -0.519740 & -2.011762 & 0.000000 \\ 1 & -1.217052 & -1.196693 & 0.000000 \\ 6 & -0.955712 & -3.268903 & 0.000000 \\ 1 & -0.280506 & -4.106561 & 0.000000 \\ 1 & -2.005687 & -3.495605 & 0.000000\end{array}$

(trans-syn)vinyl allyl fluoride $+\mathrm{LiF}$ -399.724736 au

0.115550 au

$\begin{array}{lrrr}6 & -0.383756 & -1.515622 & 0.000000 \\ 6 & 0.648527 & -0.619825 & 0.000000 \\ 6 & 0.325263 & 0.731081 & 0.000000 \\ 9 & -0.383756 & 2.709263 & 1.502694 \\ 9 & -0.383756 & 2.709263 & -1.502694 \\ 3 & -0.581482 & 3.443887 & 0.000000 \\ 1 & -0.685072 & 1.065441 & 0.000000 \\ 1 & 1.675762 & -0.930701 & 0.000000 \\ 1 & 1.066912 & 1.497487 & 0.000000 \\ 1 & -1.377938 & -1.102534 & 0.000000 \\ 6 & -0.311064 & -2.967261 & 0.000000 \\ 1 & -1.251505 & -3.487576 & 0.000000 \\ 6 & 0.824580 & -3.662628 & 0.000000 \\ 1 & 0.809560 & -4.736745 & 0.000000 \\ 1 & 1.793046 & -3.198230 & 0.000000\end{array}$

(cis-syn)vinyl allyl fluoride + LiF -399.644070 au

0.111662 au

$\begin{array}{lllll}6 & -0.390060 & -2.226960 & 0.000000\end{array}$ 


\begin{tabular}{|c|c|c|c|}
\hline 6 & -1.245810 & -1.206256 & 0.000000 \\
\hline 6 & -0.979328 & 0.301374 & 0.000000 \\
\hline 1 & -1.818619 & 1.006219 & 0.000000 \\
\hline 1 & 0.050731 & 0.675800 & 0.000000 \\
\hline 1 & -2.275869 & -1.580681 & 0.000000 \\
\hline 1 & -0.765342 & -3.256707 & 0.000000 \\
\hline 6 & 1.132486 & -2.387626 & 0.000000 \\
\hline 1 & 1.577853 & -3.389057 & 0.000000 \\
\hline 6 & 2.371465 & -1.488242 & 0.000000 \\
\hline 1 & 3.372525 & -1.934441 & 0.000000 \\
\hline 1 & 2.257355 & -0.398198 & 0.000000 \\
\hline 9 & -0.390060 & 2.318470 & -1.502694 \\
\hline 9 & -0.390060 & 2.318470 & 1.502694 \\
\hline 0 & -0.236688 & 3.063618 & 0.00000 \\
\hline
\end{tabular}

(trans)F allyl chloride $+\mathrm{LiCl}$

-1141.824828 au

$0.070430 \mathrm{au}$

$\begin{array}{lccc}6 & -0.464509 & -0.533231 & 0.000000 \\ 6 & -1.847803 & -0.668248 & 0.000000 \\ 6 & -2.550195 & 0.486581 & 0.000000 \\ 3 & 2.748590 & 0.156499 & 0.000000 \\ 17 & 1.755708 & 0.012546 & 1.972006 \\ 17 & 1.755708 & 0.012546 & -1.972006 \\ 1 & 0.000000 & 0.426366 & 0.000000 \\ 1 & 0.189292 & -1.378197 & 0.000000 \\ 1 & -2.344262 & -1.619458 & 0.000000 \\ 1 & -2.112372 & 1.467673 & 0.000000 \\ 9 & -3.833052 & 0.499661 & 0.000000\end{array}$

(cis) $\mathrm{F}$ allyl chloride $+\mathrm{LiCl}$

-1141.819776 au

$0.0705671 \mathrm{au}$

$\begin{array}{lccc}6 & -0.597022 & 0.564868 & 0.000000 \\ 6 & -0.949779 & 1.917387 & 0.000000 \\ 6 & 0.005329 & 2.871633 & 0.000000 \\ 3 & 0.264321 & -2.557484 & 0.000000 \\ 17 & 0.005329 & -1.589997 & 1.975754 \\ 17 & 0.005329 & -1.589997 & -1.975754 \\ 1 & 0.422753 & 0.253777 & 0.000000 \\ 1 & -1.341399 & -0.198685 & 0.000000 \\ 1 & -1.976165 & 2.228256 & 0.000000 \\ 9 & 1.262453 & 2.599861 & 0.000000 \\ 1 & -0.192585 & 3.926932 & 0.000000\end{array}$

(trans) $\mathrm{Cl}$ allyl chloride $+\mathrm{LiCl}$

-1501.865776 au

$0.068602 \mathrm{au}$

$\begin{array}{llll}6 & -0.196437 & -2.113986 & 0.000000\end{array}$

$\begin{array}{lllll}1 & -1.211054 & -1.761917 & 0.000000\end{array}$

Allyl SN2 SI v2a.doc

- S15- 


$\begin{array}{lccc}6 & 0.851848 & -1.259225 & 0.000000 \\ 6 & 0.545090 & 0.103544 & 0.000000 \\ 17 & -0.196437 & 2.249114 & 1.972751 \\ 17 & -0.196437 & 2.249114 & -1.972751 \\ 3 & -0.449048 & 3.218688 & 0.000000 \\ 1 & 1.305207 & 0.854003 & 0.000000 \\ 1 & -0.465114 & 0.443802 & 0.000000 \\ 1 & 1.865755 & -1.610280 & 0.000000 \\ 17 & -0.039517 & -3.790208 & 0.000000\end{array}$

(cis) $\mathrm{Cl}$ allyl chloride $+\mathrm{LiCl}$

-1501.860403 au

0.068691 au

$\begin{array}{lccc}6 & 1.153397 & -2.347546 & 0.000000 \\ 6 & 1.667494 & -1.095445 & 0.000000 \\ 6 & 0.861481 & 0.050797 & 0.000000 \\ 17 & -0.481399 & 1.838472 & 1.976782 \\ 17 & -0.481399 & 1.838472 & -1.976782 \\ 3 & -1.060037 & 2.654247 & 0.000000 \\ 1 & 1.295794 & 1.024955 & 0.000000 \\ 1 & -0.202251 & -0.008078 & 0.000000 \\ 1 & 2.736762 & -0.997579 & 0.000000 \\ 1 & 1.806905 & -3.199326 & 0.000000 \\ 17 & -0.481399 & -2.761036 & 0.000000\end{array}$

(trans) $\mathrm{CN}$ allyl chloride $+\mathrm{LiCl}$

-1134.686626 au

0.077108 au

$\begin{array}{lccc}6 & 0.888112 & 2.074276 & 0.000000 \\ 6 & -0.338525 & 1.526852 & 0.000000 \\ 6 & -0.428185 & 0.109976 & 0.000000 \\ 17 & -0.428185 & -2.028938 & 1.957892 \\ 17 & -0.428185 & -2.028938 & -1.957892 \\ 3 & -0.482782 & -3.090268 & 0.000000 \\ 1 & -1.370224 & -0.388421 & 0.000000 \\ 1 & 0.451982 & -0.488684 & 0.000000 \\ 1 & -1.226686 & 2.129294 & 0.000000 \\ 1 & 1.774037 & 1.464717 & 0.000000 \\ 6 & 1.103438 & 3.494631 & 0.000000 \\ 7 & 1.289783 & 4.614769 & 0.000000\end{array}$

(cis) $\mathrm{CN}$ allyl chloride $+\mathrm{LiCl}$

-1134.682282 au

$0.077198 \mathrm{au}$

$\begin{array}{lccl}6 & -2.598236 & -0.000639 & 0.779398 \\ 6 & -1.438919 & -0.001146 & 1.457404 \\ 6 & -0.180321 & -0.000385 & 0.790004 \\ 17 & 1.704486 & 1.962199 & -0.138946 \\ 17 & 1.704198 & -1.962101 & -0.140311 \\ 3 & 2.640915 & 0.000203 & -0.645015\end{array}$




\begin{tabular}{|c|c|c|c|}
\hline & 0.724478 & -0.000848 & 1.349353 \\
\hline & -0.117150 & 0.000331 & -0.273814 \\
\hline & -1.460784 & -0.002144 & 2.530250 \\
\hline & -3.535308 & -0.001165 & 1.306689 \\
\hline & -2.711573 & 0.000602 & -0.653720 \\
\hline & -2.843901 & 0.001564 & -1.781226 \\
\hline
\end{tabular}

(trans)Me allyl chloride $+\mathrm{LiCl}$

-1082.017571 au

$0.1076217 \mathrm{au}$

$\begin{array}{lccc}6 & -.419034 & -.548654 & .000000 \\ 6 & -1.795866 & -.743042 & .000000 \\ 6 & -2.593949 & .359318 & .000000 \\ 3 & 2.774081 & .264383 & .000000 \\ 17 & 1.835672 & .085035 & 1.988123 \\ 17 & 1.835672 & .085035 & -1.988123 \\ 1 & .000000 & .432282 & .000000 \\ 1 & .275067 & -1.361899 & .000000 \\ 1 & -2.212118 & -1.733398 & .000000 \\ 6 & -4.077775 & .352001 & .000000 \\ 1 & -2.116874 & 1.325591 & .000000 \\ 1 & -4.486725 & -.648629 & .000000 \\ 1 & -4.437343 & .891989 & .872100 \\ 1 & -4.437343 & .891989 & -.872100\end{array}$

(cis)Me allyl chloride $+\mathrm{LiCl}$

-1082.013739 au

$0.1075562 \mathrm{au}$

$\begin{array}{lccc}6 & -1.056269 & .082868 & .000000 \\ 6 & -1.929150 & 1.162720 & .000000 \\ 6 & -1.461958 & 2.446652 & .000000 \\ 3 & 1.495142 & -2.058020 & .000000 \\ 17 & .832026 & -1.363445 & 1.985316 \\ 17 & .832026 & -1.363445 & -1.985316 \\ 1 & .000000 & .202340 & .000000 \\ 1 & -1.410572 & -.927136 & .000000 \\ 1 & -2.989100 & .990355 & .000000 \\ 6 & -.057127 & 2.933025 & .000000 \\ 1 & -2.206431 & 3.226608 & .000000 \\ 1 & .689115 & 2.155533 & .000000 \\ 1 & .084857 & 3.565938 & -.872118 \\ 1 & .084857 & 3.565938 & .872118\end{array}$

(cis) $\mathrm{SH}$ allyl chloride $+\mathrm{LiCl}$

-1440.482168 au

$0.078040 \mathrm{au}$

$\begin{array}{llcc}6 & 1.149999 & -2.361306 & 0.000000 \\ 6 & 1.768624 & -1.128454 & 0.000000 \\ 6 & 1.052909 & 0.048489 & 0.000000 \\ 17 & -0.527296 & 1.845110 & 1.994062\end{array}$

Allyl SN2 SI v2a.doc - - S17- 


$\begin{array}{lccc}17 & -0.527296 & 1.845110 & -1.994062 \\ 3 & -1.059176 & 2.612378 & 0.000000 \\ 1 & 1.539401 & 1.000930 & 0.000000 \\ 1 & -0.013694 & 0.073022 & 0.000000 \\ 1 & 2.841955 & -1.110020 & 0.000000 \\ 1 & 1.782068 & -3.231508 & 0.000000 \\ 16 & -0.527296 & -2.666736 & 0.000000 \\ 1 & -0.436605 & -3.987902 & 0.000000\end{array}$

(trans) $\mathrm{OH}$ allyl chloride $+\mathrm{LiCl}$

-1117.823565 au

0.083192 au

$\begin{array}{lccc}6 & -2.641340 & 0.615327 & 0.000000 \\ 6 & -1.881282 & -0.533731 & 0.000000 \\ 6 & -0.509382 & -0.456119 & 0.000000 \\ 17 & 1.859363 & -0.082553 & 2.004307 \\ 17 & 1.859363 & -0.082553 & -2.004307 \\ 3 & 2.761958 & 0.003290 & 0.000000 \\ 1 & 0.101569 & -1.333205 & 0.000000 \\ 1 & 0.000000 & 0.481586 & 0.000000 \\ 1 & -2.396156 & -1.474489 & 0.000000 \\ 8 & -4.068961 & 0.532877 & 0.000000 \\ 1 & -2.210834 & 1.591761 & 0.000000 \\ 1 & -4.255076 & 1.515405 & 0.000000\end{array}$

(cis) $\mathrm{OH}$ allyl chloride $+\mathrm{LiCl}$

-1117.841604 au 0.084059 au

$\begin{array}{lcll}6 & 0.022457 & 2.908086 & 0.000000 \\ 6 & -0.996568 & 1.980935 & 0.000000 \\ 6 & -0.709658 & 0.637128 & 0.000000 \\ 17 & 0.022457 & -1.646403 & 2.004307 \\ 17 & 0.022457 & -1.646403 & -2.004307 \\ 3 & 0.245590 & -2.525185 & 0.000000 \\ 1 & -1.482771 & -0.101003 & 0.000000 \\ 1 & 0.295025 & 0.277445 & 0.000000 \\ 1 & -2.005110 & 2.345577 & 0.000000 \\ 1 & -0.203794 & 3.961707 & 0.000000 \\ 8 & 1.266132 & 2.575346 & 0.000000 \\ 1 & 1.869913 & 3.309878 & 0.000000\end{array}$

(trans-anti)vinyl allyl chloride $+\mathrm{LiCl}$

-1119.871302 au

0.113953 au

$\begin{array}{lccc}6 & 0.293400 & -2.182842 & 0.000000 \\ 6 & 1.251575 & -1.198060 & 0.000000 \\ 6 & 0.823918 & 0.112915 & 0.000000 \\ 17 & -0.445569 & 2.193563 & 2.000711 \\ 17 & -0.445569 & 2.193563 & -2.000711 \\ 3 & -0.811762 & 3.030691 & 0.000000 \\ 1 & 1.502478 & 0.941007 & 0.000000\end{array}$




$\begin{array}{lrrr}1 & -0.214686 & 0.356582 & 0.000000 \\ 1 & 2.297392 & -1.443800 & 0.000000 \\ 1 & -0.739969 & -1.878953 & 0.000000 \\ 6 & 0.559149 & -3.596060 & 0.000000 \\ 1 & 1.582991 & -3.923868 & 0.000000 \\ 6 & -0.445569 & -4.474606 & 0.000000 \\ 1 & -1.473752 & -4.158821 & 0.000000 \\ 1 & -0.264661 & -5.533469 & 0.000000\end{array}$

(trans-syn)vinyl allyl chloride $+\mathrm{LiCl}$

-1119.86540 au

0.113913 au

$\begin{array}{llll}6 & 1.031401 & 1.990664 & 0.000000 \\ 6 & -0.269936 & 1.548675 & 0.000000 \\ 6 & -0.478708 & 0.185203 & 0.000000 \\ 17 & -0.478708 & -2.224122 & 2.000341 \\ 17 & -0.478708 & -2.224122 & -2.000341 \\ 3 & -0.564452 & -3.136557 & 0.000000 \\ 1 & -1.458494 & -0.244297 & 0.000000 \\ 1 & 0.336831 & -0.503244 & 0.000000 \\ 1 & -1.103205 & 2.224477 & 0.000000 \\ 1 & 1.795125 & 1.231788 & 0.000000 \\ 6 & 1.510241 & 3.357122 & 0.000000 \\ 1 & 2.576892 & 3.486763 & 0.000000 \\ 6 & 0.715077 & 4.427769 & 0.000000 \\ 1 & 1.130495 & 5.418355 & 0.000000 \\ 1 & -0.356678 & 4.359377 & 0.000000\end{array}$

(trans) F allyl fluoride $+\mathrm{NaF}$

-576.054066 au

$0.0708054 \mathrm{au}$

$\begin{array}{llll}6 & .000000 & .455061 & .000000 \\ 6 & 1.259864 & 1.081321 & .000000 \\ 6 & 2.323264 & .265846 & .000000 \\ 11 & -2.636754 & -1.006189 & .000000 \\ 9 & -1.499088 & -.441115 & 1.631104 \\ 9 & -1.499088 & -.441115 & -1.631104 \\ 1 & -.083761 & -.600924 & .000000 \\ 1 & -.898953 & 1.018212 & .000000 \\ 1 & 1.377465 & 2.147907 & .000000 \\ 9 & 3.535863 & .715098 & .000000 \\ 1 & 2.271596 & -.806288 & .000000\end{array}$

(cis)F allyl fluoride $+\mathrm{NaF}$

-576.049058 au

$0.0709671 \mathrm{au}$

$\begin{array}{lccc}6 & -.248631 & -.565900 & .000000 \\ 6 & -1.600558 & -.985861 & .000000 \\ 6 & -2.597888 & -.092411 & .000000 \\ 11 & 2.586633 & .316200 & .000000\end{array}$

Allyl SN2 SI v2a.doc

- S19- 


$\begin{array}{cccc}9 & 1.377529 & -.062089 & 1.638952 \\ 9 & 1.377529 & -.062089 & -1.638952 \\ 1 & .000000 & .463329 & .000000 \\ 1 & .534830 & -1.277995 & .000000 \\ 1 & -1.856753 & -2.027426 & .000000 \\ 9 & -2.400370 & 1.188031 & .000000 \\ 1 & -3.640770 & -.345761 & .000000\end{array}$

(trans) $\mathrm{Cl}$ allyl fluoride $+\mathrm{NaF}$ $-936.095040 \mathrm{au}$ 0.069026 au

$\begin{array}{lccc}6 & -0.175422 & -1.821443 & 0.000000 \\ 1 & -1.213312 & -1.548874 & 0.000000 \\ 6 & 0.800258 & -0.902252 & 0.000000 \\ 6 & 0.399863 & 0.455966 & 0.000000 \\ 9 & -0.175422 & 2.080904 & 1.631989 \\ 9 & -0.175422 & 2.080904 & -1.631989 \\ 11 & -0.529909 & 3.303298 & 0.000000 \\ 1 & 1.114655 & 1.238997 & 0.000000 \\ 1 & -0.626236 & 0.717961 & 0.000000 \\ 1 & 1.837217 & -1.178396 & 0.000000 \\ 17 & 0.101534 & -3.495051 & 0.000000\end{array}$

(cis) $\mathrm{Cl}$ allyl fluoride $+\mathrm{NaF}$ -936.089579 au 0.069155 au

$\begin{array}{lccc}6 & 1.218739 & -1.998400 & 0.000000 \\ 6 & 1.607244 & -0.714833 & 0.000000 \\ 6 & 0.694717 & 0.375313 & 0.000000 \\ 9 & -0.381749 & 1.676996 & 1.638884 \\ 9 & -0.381749 & 1.676996 & -1.638884 \\ 11 & -1.188757 & 2.658775 & 0.000000 \\ 1 & 1.057735 & 1.369634 & 0.000000 \\ 1 & -0.351236 & 0.214803 & 0.000000 \\ 1 & 2.663024 & -0.517517 & 0.000000 \\ 1 & 1.943828 & -2.789360 & 0.000000 \\ 17 & -0.381749 & -2.569559 & 0.000000\end{array}$

(trans) $\mathrm{CN}$ allyl fluoride $+\mathrm{NaF}$ -568.919920 au $0.077683 \mathrm{au}$

$\begin{array}{lccc}6 & -0.186493 & -1.958310 & 0.000000 \\ 6 & 0.755931 & -1.012653 & 0.000000 \\ 6 & 0.335803 & 0.362764 & 0.000000 \\ 9 & -0.186493 & 1.882757 & 1.627806 \\ 9 & -0.186493 & 1.882757 & -1.627806 \\ 11 & -0.561376 & 3.138411 & 0.000000 \\ 1 & 1.052907 & 1.140384 & 0.000000 \\ 1 & -0.695998 & 0.593192 & 0.000000 \\ 1 & 1.801121 & -1.257185 & 0.000000\end{array}$




$\begin{array}{rrrr}1 & -1.231861 & -1.708313 & 0.000000 \\ 6 & 0.122492 & -3.364730 & 0.000000 \\ 7 & 0.348493 & -4.477522 & 0.000000\end{array}$

(cis) $\mathrm{CN}$ allyl fluoride $+\mathrm{NaF}$ -568.916010 au 0.077911 au

$\begin{array}{lccc}6 & -0.476833 & -2.391363 & 0.000000 \\ 6 & 0.671986 & -1.711570 & 0.000000 \\ 6 & 0.709196 & -0.267554 & 0.000000 \\ 9 & 0.778584 & 1.283716 & 1.633085 \\ 9 & 0.778584 & 1.283716 & -1.633085 \\ 11 & 0.778584 & 2.598754 & 0.000000 \\ 1 & 1.643274 & 0.224322 & 0.000000 \\ 1 & -0.194509 & 0.281220 & 0.000000 \\ 1 & 1.598846 & -2.252810 & 0.000000 \\ 1 & -0.476035 & -3.466273 & 0.000000 \\ 6 & -1.786458 & -1.790049 & 0.000000 \\ 7 & -2.836839 & -1.359491 & 0.000000\end{array}$

(trans)Me allyl fluoride $+\mathrm{NaF}$ -516.243800 au

0.108103 au

$\begin{array}{llll}6 & .000000 & .432667 & .000000 \\ 6 & 1.312194 & .943241 & .000000 \\ 6 & 2.340426 & .069532 & .000000 \\ 11 & -2.771250 & -.770422 & .000000 \\ 9 & -1.620882 & -.319268 & 1.648369 \\ 9 & -1.620882 & -.319268 & -1.648369 \\ 1 & -.176444 & -.612763 & .000000 \\ 1 & -.849030 & 1.070001 & .000000 \\ 1 & 1.481332 & 2.004635 & .000000 \\ 6 & 3.784672 & .429281 & .000000 \\ 1 & 2.108128 & -.982693 & .000000 \\ 1 & 3.939977 & 1.499672 & .000000 \\ 1 & 4.265959 & -.002850 & .873005 \\ 1 & 4.265959 & -.002850 & -.873005\end{array}$

(cis)Me allyl fluoride $+\mathrm{NaF}$ -516.239759 au $0.108200 \mathrm{au}$

$\begin{array}{lccc}6 & -.155555 & -.711390 & .000000 \\ 6 & -1.442158 & -1.284161 & .000000 \\ 6 & -2.562618 & -.525771 & .000000 \\ 11 & 2.559502 & .638083 & .000000 \\ 9 & 1.419517 & .152311 & 1.645688 \\ 9 & 1.419517 & .152311 & -1.645688 \\ 1 & .000000 & .334047 & .000000 \\ 1 & .713838 & -1.321835 & .000000 \\ 1 & -1.534475 & -2.354483 & .000000\end{array}$

Allyl SN2 SI v2a.doc - S21- 


\begin{tabular}{lrrr}
6 & -2.690772 & .959964 & .000000 \\
1 & -3.502500 & -1.054534 & .000000 \\
1 & -1.749033 & 1.484626 & .000000 \\
1 & -3.263515 & 1.259905 & -.873044 \\
1 & -3.263515 & 1.259905 & .873044 \\
\multicolumn{5}{c}{} \\
(trans)SH allyl fluoride + NaF \\
-874.713870 au \\
0.078420 au \\
6 \\
6 & -0.166021 & -1.831495 & 0.000000 \\
6 & 0.839758 & -0.919425 & 0.000000 \\
6 & 0.480301 & 0.431004 & 0.000000 \\
9 & -0.166021 & 2.140229 & 1.648463 \\
9 & -0.166021 & 2.140229 & -1.648463 \\
11 & -0.488134 & 3.326020 & 0.000000 \\
1 & 1.209076 & 1.204833 & 0.000000 \\
1 & -0.536937 & 0.730370 & 0.000000 \\
1 & 1.871481 & -1.219242 & 0.000000 \\
1 & -1.183820 & -1.485751 & 0.000000 \\
16 & 0.079399 & -3.533280 & 0.000000 \\
1 & -1.196567 & -3.888572 & 0.000000
\end{tabular}

(cis) $\mathrm{SH}$ allyl fluoride $+\mathrm{NaF}$ -874.707051 au 0.078303 au

$\begin{array}{lccc}6 & 1.217523 & -2.007822 & 0.000000 \\ 6 & 1.653518 & -0.719462 & 0.000000 \\ 6 & 0.776377 & 0.372632 & 0.000000 \\ 9 & -0.418280 & 1.721003 & 1.647321 \\ 9 & -0.418280 & 1.721003 & -1.647321 \\ 11 & -1.187540 & 2.687827 & 0.000000 \\ 1 & 1.143545 & 1.368657 & 0.000000 \\ 1 & -0.277523 & 0.253694 & 0.000000 \\ 1 & 2.713613 & -0.548485 & 0.000000 \\ 1 & 1.958331 & -2.787225 & 0.000000 \\ 16 & -0.418280 & -2.553462 & 0.000000 \\ 1 & -0.138016 & -3.847503 & 0.000000\end{array}$

(trans) $\mathrm{OH}$ allyl fluoride $+\mathrm{NaF}$ -552.072236 au 0.084236 au

\begin{tabular}{|c|c|c|c|}
\hline 6 & -0.234577 & -2.318610 & 0.000000 \\
\hline 6 & 0.857562 & -1.502217 & 0.000000 \\
\hline 6 & 0.594315 & -0.145068 & 0.000000 \\
\hline 9 & -0.132997 & 1.612447 & 1.654185 \\
\hline 9 & -0.132997 & 1.612447 & -1.654185 \\
\hline 11 & -0.406392 & 2.792589 & 0.000000 \\
\hline 1 & 1.370774 & 0.584342 & 0.000000 \\
\hline 1 & -0.396853 & 0.232567 & 0.000000 \\
\hline 1 & 1.848963 & -1.913175 & 0.000000 \\
\hline
\end{tabular}




\begin{tabular}{|c|c|c|c|}
\hline 1 & -1.226528 & -1.899626 & 0.000000 \\
\hline 8 & -0.132997 & -3.611708 & 0.000000 \\
\hline 1 & -0.971905 & -4.057594 & 0.0 \\
\hline
\end{tabular}

(cis) $\mathrm{OH}$ allyl fluoride $+\mathrm{NaF}$ -552.064992 au

0.084261 au

$\begin{array}{lccc}6 & 2.624909 & -0.000237 & 0.144965 \\ 6 & 1.600269 & -0.000750 & 1.041755 \\ 6 & 0.274989 & -0.000048 & 0.613334 \\ 9 & -1.455529 & -1.664144 & 0.018766 \\ 9 & -1.456070 & 1.664166 & 0.020185 \\ 11 & -2.601692 & -0.000070 & -0.348650 \\ 1 & -0.527124 & -0.000583 & 1.308741 \\ 1 & 0.025042 & 0.000843 & -0.417846 \\ 1 & 1.848683 & -0.001802 & 2.085225 \\ 1 & 3.645720 & -0.000862 & 0.489617 \\ 8 & 2.446666 & 0.000988 & -1.142958 \\ 1 & 3.256347 & 0.001295 & -1.637800\end{array}$

(trans-anti)vinyl allyl fluoride $+\mathrm{NaF}$ -554.095228 au

0.114316 au

$\begin{array}{lrcc}6 & 0.194251 & -1.895245 & 0.000000 \\ 6 & 1.051679 & -0.842505 & 0.000000 \\ 6 & 0.516575 & 0.451703 & 0.000000 \\ 9 & -0.314973 & 2.079301 & 1.654366 \\ 9 & -0.314973 & 2.079301 & -1.654366 \\ 11 & -0.786661 & 3.203402 & 0.000000 \\ 1 & 1.134364 & 1.316195 & 0.000000 \\ 1 & -0.532241 & 0.610720 & 0.000000 \\ 1 & 2.115749 & -0.993412 & 0.000000 \\ 1 & -0.863107 & -1.690079 & 0.000000 \\ 6 & 0.589462 & -3.288962 & 0.000000 \\ 1 & 1.641515 & -3.512594 & 0.000000 \\ 6 & -0.314973 & -4.264722 & 0.000000 \\ 1 & -1.370743 & -4.058220 & 0.000000 \\ 1 & -0.024723 & -5.299062 & 0.000000\end{array}$

(cis-anti)vinyl allyl fluoride $+\mathrm{NaF}$ -554.090825 au

$0.114350 \mathrm{au}$

\begin{tabular}{|c|c|c|c|}
\hline 6 & 1.061577 & -2.036016 & 0.000000 \\
\hline 6 & 1.624277 & -0.795045 & 0.000000 \\
\hline 6 & 0.846813 & 0.369464 & 0.000000 \\
\hline 9 & -0.347415 & 1.778116 & 1.648617 \\
\hline 9 & -0.347415 & 1.778116 & -1.648617 \\
\hline 11 & -1.014914 & 2.805150 & 0.000000 \\
\hline 1 & 1.299126 & 1.332166 & 0.000000 \\
\hline 1 & -0.210773 & 0.352729 & 0.000000 \\
\hline
\end{tabular}




$\begin{array}{llll}1 & 2.694971 & -0.711512 & 0.000000 \\ 1 & 1.734609 & -2.877842 & 0.000000 \\ 6 & -0.347415 & -2.379006 & 0.000000 \\ 1 & -1.081166 & -1.596581 & 0.000000 \\ 6 & -0.725612 & -3.654718 & 0.000000 \\ 1 & -0.012914 & -4.460717 & 0.000000 \\ 1 & -1.764158 & -3.929060 & 0.000000\end{array}$

(trans-syn)vinyl allyl fluoride $+\mathrm{NaF}$ -554.089547 au

0.114223 au

$\begin{array}{lrcc}6 & -1.863765 & -0.146923 & 0.552578 \\ 6 & -1.075674 & -0.047710 & -0.548649 \\ 6 & 0.312217 & -0.012246 & -0.362768 \\ 9 & 2.150166 & -1.627641 & -0.124629 \\ 9 & 2.046592 & 1.670630 & 0.109095 \\ 11 & 3.307271 & 0.047799 & 0.158613 \\ 1 & 0.985749 & 0.072867 & -1.179736 \\ 1 & 0.737258 & -0.069374 & 0.607551 \\ 1 & -1.485943 & -0.007866 & -1.539942 \\ 1 & -1.366041 & -0.221683 & 1.504413 \\ 6 & -3.321424 & -0.177034 & 0.599720 \\ 1 & -3.757019 & -0.513321 & 1.523654 \\ 6 & -4.110968 & 0.221780 & -0.392719 \\ 1 & -5.179953 & 0.194830 & -0.289563 \\ 1 & -3.727158 & 0.604660 & -1.320285\end{array}$

(cis-syn)vinyl allyl fluoride $+\mathrm{NaF}$ -554.0110233 au 0.110277 au

$\begin{array}{lrrr}6 & 2.391354 & -0.977421 & 0.009011 \\ 6 & 1.178900 & -1.528831 & 0.015970 \\ 6 & -0.202463 & -0.868698 & 0.011337 \\ 1 & -1.106169 & -1.488762 & 0.018563 \\ 1 & -0.287636 & 0.223937 & 0.000796 \\ 1 & 1.264073 & -2.621465 & 0.026511 \\ 1 & 3.283147 & -1.614520 & 0.014058 \\ 6 & 2.953532 & 0.446554 & -0.005606 \\ 1 & 4.037614 & 0.607720 & -0.008593 \\ 6 & 2.418439 & 1.880938 & -0.018892 \\ 1 & 3.116212 & 2.726068 & -0.028043 \\ 1 & 1.337606 & 2.062645 & -0.019252 \\ 9 & -1.793101 & -0.006888 & -1.653421 \\ 9 & -1.792554 & 0.045060 & 1.654334 \\ 11 & -2.892048 & 0.549597 & -0.007561\end{array}$

\section{Reactants}

$\mathrm{Cl}^{-}$

$-459.539661 \mathrm{au}$

$\mathrm{LiF}$

$-106.946363 \mathrm{au}$ 


\begin{tabular}{|c|c|c|c|}
\hline \multirow{2}{*}{\multicolumn{4}{|c|}{$0.002114 \mathrm{au}$}} \\
\hline & & $\mathrm{LiCl}$ & \\
\hline \multicolumn{4}{|c|}{$-467.011399 \mathrm{au}$} \\
\hline \multicolumn{4}{|c|}{$0.001416 \mathrm{au}$} \\
\hline \multicolumn{4}{|c|}{$\mathrm{NaF}$} \\
\hline \multicolumn{4}{|c|}{$-261.321512 a u$} \\
\hline \multicolumn{4}{|c|}{$0.001239 \mathrm{au}$} \\
\hline \multicolumn{4}{|c|}{ Allyl fluoride } \\
\hline \multicolumn{4}{|c|}{-215.928943 au } \\
\hline \multicolumn{4}{|c|}{$0.078642 \mathrm{au}$} \\
\hline \multicolumn{4}{|c|}{ Allyl chloride } \\
\hline \multicolumn{4}{|c|}{-575.976092 au } \\
\hline \multicolumn{4}{|c|}{$0.077036 \mathrm{au}$} \\
\hline \multicolumn{4}{|c|}{ 2-F allyl fluoride } \\
\hline \multicolumn{4}{|c|}{-314.784459 au } \\
\hline \multicolumn{4}{|c|}{$0.070751 \mathrm{au}$} \\
\hline 6 & -1.550328 & -0.829753 & -0.192192 \\
\hline 6 & -0.542536 & -0.034539 & 0.082134 \\
\hline 6 & 0.837865 & -0.434264 & 0.482633 \\
\hline 1 & 1.104614 & 0.022125 & 1.426142 \\
\hline 1 & 0.915268 & -1.508518 & 0.554385 \\
\hline 1 & -2.517914 & -0.435746 & -0.439439 \\
\hline 1 & -1.418365 & -1.894240 & -0.178654 \\
\hline 9 & -0.694404 & 1.290421 & 0.050788 \\
\hline 9 & 1.744004 & -0.000675 & -0.450553 \\
\hline \multicolumn{4}{|c|}{ 2-Me allyl fluoride } \\
\hline \multicolumn{4}{|c|}{$-254.968371 \mathrm{au}$} \\
\hline \multicolumn{4}{|c|}{$0.108751 \mathrm{au}$} \\
\hline 6 & -1.456983 & -1.027512 & -0.184644 \\
\hline 1 & -1.224140 & -2.078345 & -0.182015 \\
\hline 1 & -2.476421 & -0.762841 & -0.404320 \\
\hline 6 & -0.539089 & -0.108496 & 0.069768 \\
\hline 6 & 0.862678 & -0.513279 & 0.431313 \\
\hline 1 & 1.012936 & -1.577932 & 0.328744 \\
\hline 1 & 1.109790 & -0.208019 & 1.440865 \\
\hline 9 & 1.770053 & 0.114120 & -0.398873 \\
\hline 6 & -0.817846 & 1.372469 & 0.056659 \\
\hline 1 & -0.197331 & 1.871560 & -0.679689 \\
\hline 1 & -0.590998 & 1.816273 & 1.023091 \\
\hline 1 & -1.856870 & 1.573139 & -0.175391 \\
\hline \multicolumn{4}{|c|}{ 2-F allyl chloride } \\
\hline \multicolumn{4}{|c|}{$-674.831510 \mathrm{au}$} \\
\hline \multicolumn{4}{|c|}{$0.069016 \mathrm{au}$} \\
\hline 6 & -1.923169 & -0.823057 & -0.351876 \\
\hline 6 & -0.973771 & -0.034699 & 0.096936 \\
\hline 6 & 0.310974 & -0.446093 & 0.728116 \\
\hline 1 & 0.441420 & 0.050646 & 1.677125 \\
\hline 1 & 0.338495 & -1.513548 & 0.866516 \\
\hline 1 & -2.827696 & -0.419071 & -0.765039 \\
\hline 1 & -1.805398 & -1.888504 & -0.316086 \\
\hline 9 & -1.114650 & 1.290105 & 0.046126 \\
\hline
\end{tabular}




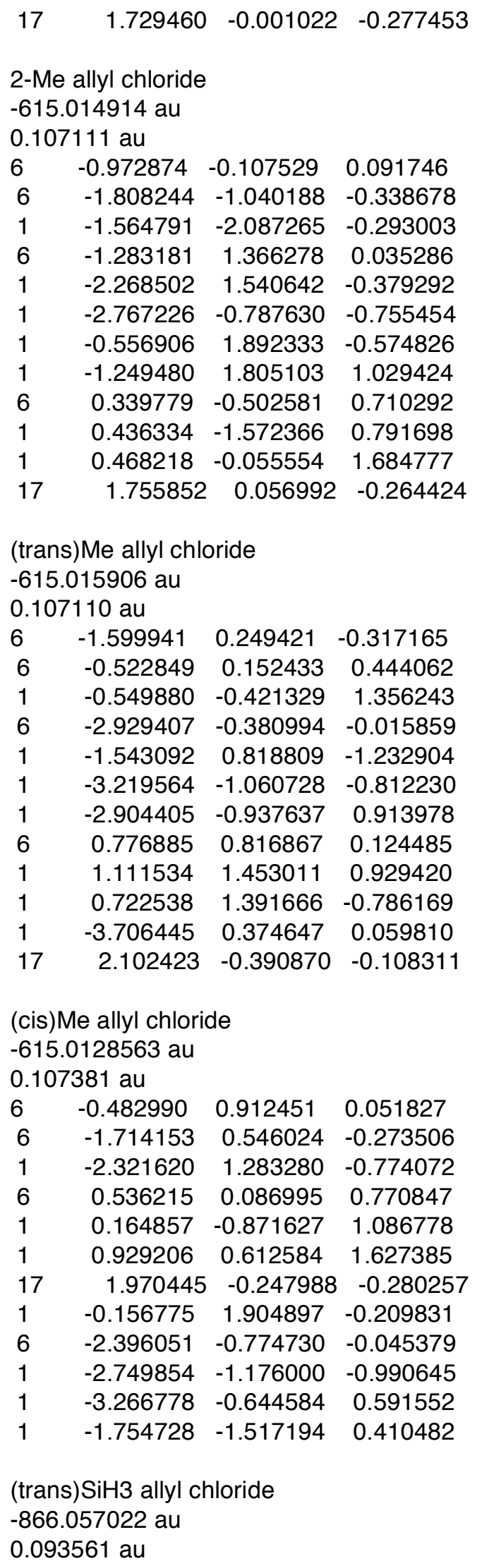




$\begin{array}{lccc}6 & -1.063422 & 0.487478 & -0.320838 \\ 6 & 0.012291 & 0.202889 & 0.403365 \\ 1 & -0.042207 & -0.496095 & 1.222562 \\ 1 & -0.950658 & 1.186230 & -1.138794 \\ 6 & 1.361549 & 0.815622 & 0.181934 \\ 1 & 1.699234 & 1.350705 & 1.056843 \\ 1 & 1.368038 & 1.477182 & -0.669159 \\ 17 & 2.607990 & -0.449231 & -0.126355 \\ 14 & -2.759226 & -0.254484 & -0.009395 \\ 1 & -3.738449 & 0.810261 & 0.284723 \\ 1 & -3.226885 & -0.987258 & -1.201708 \\ 1 & -2.678242 & -1.177261 & 1.138341\end{array}$

(cis) $\mathrm{SiH} 3$ allyl chloride -866.053392 au 0.093779 au

$\begin{array}{llll}6 & -1.171954 & 0.954071 & -0.234032 \\ 1 & -1.618747 & 1.829341 & -0.685832 \\ 6 & 0.112460 & 1.076408 & 0.084449 \\ 1 & 0.623424 & 2.002407 & -0.127273 \\ 6 & 0.971548 & 0.044444 & 0.748864 \\ 1 & 0.452877 & -0.878163 & 0.940340 \\ 1 & 1.381571 & 0.422212 & 1.673448 \\ 14 & -2.352570 & -0.499033 & -0.043679 \\ 1 & -3.438378 & -0.127143 & 0.883968 \\ 1 & -2.949731 & -0.801684 & -1.357440 \\ 1 & -1.680637 & -1.705051 & 0.471156 \\ 17 & 2.393722 & -0.364999 & -0.281326\end{array}$

(trans)F allyl chloride

-674.829118 au

0.0694014 au

$\begin{array}{lccc}6 & 0.581154 & 0.135702 & 0.464755 \\ 6 & 1.629756 & 0.179954 & -0.323442 \\ 1 & 1.685162 & 0.667933 & -1.276856 \\ 6 & -0.699663 & 0.814942 & 0.110172 \\ 1 & -0.623532 & 1.365431 & -0.814253 \\ 1 & 0.635294 & -0.397311 & 1.397061 \\ 1 & -1.022526 & 1.482845 & 0.893001 \\ 17 & -2.043310 & -0.368325 & -0.102715 \\ 9 & 2.777154 & -0.404551 & 0.004255\end{array}$

(cis)F allyl chloride

-674.8299012 au

0.069668 au

$\begin{array}{lccc}6 & -0.566477 & 0.833364 & 0.135182 \\ 6 & -1.770947 & 0.460382 & -0.231998 \\ 6 & 0.457242 & -0.072308 & 0.737888 \\ 1 & 0.097322 & -1.082238 & 0.832354 \\ 1 & -0.304728 & 1.865776 & -0.006772 \\ 1 & 0.779104 & 0.287350 & 1.702694 \\ 17 & 1.943735 & -0.138690 & -0.279281 \\ 1 & -2.517338 & 1.101843 & -0.654472\end{array}$




\begin{tabular}{lccc}
9 & -2.201863 & -0.793736 & -0.108051 \\
\multicolumn{5}{c}{ (trans) Cl allyl chloride } \\
-1034.8750504 au \\
0.067807 au \\
6 & 0.058338 & 0.201402 & 0.446223 \\
6 & 1.104365 & 0.404415 & -0.325790 \\
1 & 1.080676 & 0.999239 & -1.217691 \\
6 & -1.272000 & 0.820582 & 0.157728 \\
1 & -1.247175 & 1.439455 & -0.724779 \\
1 & 0.134853 & -0.416336 & 1.323123 \\
17 & 2.666927 & -0.269094 & -0.001388 \\
1 & -1.620144 & 1.406556 & 0.994198 \\
17 & -2.531187 & -0.436042 & -0.118837 \\
\multicolumn{5}{c}{} \\
(cis)Cl allyl chloride & & \\
-1034.874723 au & & \\
0.068066 au & & \\
6 & -1.269100 & 0.898048 & -0.208114 \\
1 & -1.830441 & 1.713961 & -0.615679 \\
6 & -0.002983 & 1.017930 & 0.134779 \\
1 & 0.451023 & 1.981404 & -0.019612 \\
6 & 0.870993 & -0.040126 & 0.727156 \\
1 & 0.375736 & -0.992165 & 0.798914 \\
1 & 1.225755 & 0.256156 & 1.702450 \\
17 & 2.342177 & -0.285316 & -0.280214 \\
17 & -2.213679 & -0.550829 & -0.060315
\end{tabular}

(trans) CN allyl chloride

$-667.711574 \mathrm{au}$

0.076461 au

$\begin{array}{lccc}6 & 1.239467 & 0.496918 & -0.320544 \\ 6 & 0.188586 & 0.168296 & 0.414079 \\ 1 & 0.279115 & -0.565874 & 1.195109 \\ 6 & -1.158508 & 0.789546 & 0.227711 \\ 1 & -1.166546 & 1.496503 & -0.586172 \\ 1 & -1.484334 & 1.279849 & 1.132494 \\ 17 & -2.391436 & -0.461996 & -0.137780 \\ 1 & 1.179871 & 1.217387 & -1.115851 \\ 6 & 2.538880 & -0.088928 & -0.101698 \\ 7 & 3.570823 & -0.538419 & 0.057055\end{array}$

(cis)CN allyl chloride

-667.711293 au

0.076678 au

$\begin{array}{lccl}6 & -0.084090 & 1.047783 & 0.149508 \\ 6 & -1.360953 & 1.004487 & -0.201077 \\ 6 & 0.710391 & -0.096519 & 0.696251 \\ 1 & 0.162563 & -1.023773 & 0.681995 \\ 1 & 0.439147 & 1.982098 & 0.050370 \\ 1 & 1.033915 & 0.114348 & 1.704594 \\ 17 & 2.201622 & -0.338493 & -0.271818 \\ 1 & -1.862829 & 1.879878 & -0.568074\end{array}$

Allyl SN2 SI v2a.doc

- S28- 


\begin{tabular}{lccc}
6 & -2.171102 & -0.186985 & -0.118958 \\
7 & -2.823692 & -1.115826 & -0.057476 \\
\multicolumn{5}{l}{} \\
(trans) OH allyl chloride \\
-650.832121 au \\
$0.082267 \mathrm{au}$ \\
6 & 0.562768 & 0.203048 & 0.479477 \\
1 & 0.615580 & -0.199011 & 1.475820 \\
6 & 1.618015 & 0.138083 & -0.310125 \\
6 & -0.717367 & 0.824859 & 0.039617 \\
1 & -0.635171 & 1.278999 & -0.935428 \\
1 & -1.070832 & 1.561895 & 0.743068 \\
17 & -2.060010 & -0.386833 & -0.081819 \\
1 & 1.598928 & 0.514295 & -1.319312 \\
8 & 2.788749 & -0.394584 & 0.097285 \\
1 & 3.421180 & -0.419286 & -0.605335
\end{tabular}

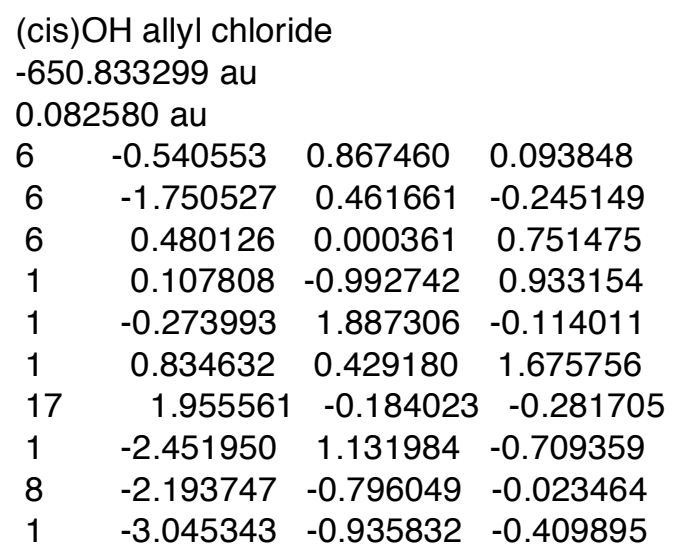

\begin{tabular}{|c|c|c|c|}
\hline \multicolumn{4}{|c|}{$\begin{array}{l}\text { (trans)SH allyl chl } \\
-973.484745 \mathrm{au} \\
0.077026 \mathrm{au}\end{array}$} \\
\hline 6 & 0.037860 & 0.184027 & 0.472986 \\
\hline 1 & 0.096938 & -0.472695 & 1.324479 \\
\hline 6 & 1.095807 & 0.416767 & -0.284211 \\
\hline 1 & 1.025777 & 1.063712 & -1.141586 \\
\hline 6 & -1.293202 & 0.811011 & 0.211927 \\
\hline 1 & -1.265197 & 1.480428 & -0.632711 \\
\hline 1 & -1.660059 & 1.342305 & 1.076466 \\
\hline 17 & -2.551768 & -0.431931 & -0.155107 \\
\hline 16 & 2.727181 & -0.223168 & -0.086263 \\
\hline . & 2.504915 & -0.971061 & 0.986156 \\
\hline
\end{tabular}

(cis)SH allyl chloride

$\begin{array}{lrll}-973.482145 \mathrm{au} & & \\ 0.077266 \mathrm{au} & & \\ 6 & -0.014744 & 1.265866 & -0.085597 \\ 1 & 0.304597 & 2.212447 & -0.486005 \\ 6 & -1.247184 & 0.868817 & -0.362423 \\ 6 & 0.988209 & 0.526923 & 0.741054 \\ 1 & 0.526139 & -0.114405 & 1.473344\end{array}$




\begin{tabular}{lccc}
1 & 1.650309 & 1.211985 & 1.244657 \\
17 & 2.059109 & -0.536800 & -0.258111 \\
1 & -1.889371 & 1.515966 & -0.931662 \\
16 & -2.059961 & -0.640791 & 0.070259 \\
1 & -0.994832 & -1.417368 & 0.205213 \\
\multicolumn{5}{c}{} \\
(trans-anti)vinyl allyl chloride \\
-652.866367 au \\
$0.113045 \mathrm{au}$ \\
6 & -0.001504 & 0.073045 & 0.573702 \\
1 & -0.035113 & -0.660898 & 1.362016 \\
6 & 1.135884 & 0.318653 & -0.063174 \\
6 & -1.283941 & 0.776239 & 0.273943 \\
1 & -1.169343 & 1.506312 & -0.511132 \\
1 & -1.692505 & 1.254612 & 1.150868 \\
17 & -2.559571 & -0.379246 & -0.276833 \\
1 & 1.155554 & 1.046226 & -0.859500 \\
1 & 2.387300 & -1.078939 & 1.034700 \\
6 & 2.406574 & -0.346277 & 0.244483 \\
1 & 4.453863 & -0.607598 & -0.132391 \\
6 & 3.544452 & -0.095213 & -0.388206 \\
1 & 3.604153 & 0.628791 & -1.182889
\end{tabular}

(trans-syn)vinyl allyl chloride -652.861455 au

0.112986 au

$\begin{array}{cccc}6 & 0.141542 & -0.112768 & 0.293764 \\ 1 & 0.294481 & -1.177855 & 0.252995 \\ 6 & 1.117481 & 0.718459 & -0.041923 \\ 6 & -1.208365 & 0.345851 & 0.740259 \\ 1 & -1.286234 & 1.421179 & 0.751284 \\ 1 & -1.457974 & -0.040356 & 1.716766 \\ 17 & -2.509856 & -0.255693 & -0.358319 \\ 1 & 0.921371 & 1.779659 & -0.054222 \\ 6 & 2.480032 & 0.313304 & -0.442381 \\ 1 & 2.920676 & 0.888399 & -1.241037 \\ 6 & 3.185097 & -0.661771 & 0.111629 \\ 1 & 4.168945 & -0.908417 & -0.244811 \\ 1 & 2.811565 & -1.234272 & 0.942360\end{array}$

(cis-anti)vinyl allyl chloride -652.863077 au

0.113381 au

$\begin{array}{cccl}6 & 0.076572 & 1.020667 & 0.074878 \\ 1 & 0.498835 & 1.963143 & -0.228581 \\ 6 & -1.206927 & 0.791396 & -0.182963 \\ 6 & 1.028635 & 0.099982 & 0.769001 \\ 1 & 0.571537 & -0.811062 & 1.112373 \\ 1 & 1.502742 & 0.591705 & 1.604550 \\ 17 & 2.379395 & -0.390607 & -0.326628 \\ 1 & -1.759403 & 1.576233 & -0.673058 \\ 6 & -1.981501 & -0.419707 & 0.116745 \\ 1 & -1.472772 & -1.251129 & 0.571591\end{array}$




$\begin{array}{rrrr}6 & -3.273960 & -0.535234 & -0.158174 \\ 1 & -3.816896 & -1.435494 & 0.065164 \\ 1 & -3.830671 & 0.264301 & -0.616284\end{array}$

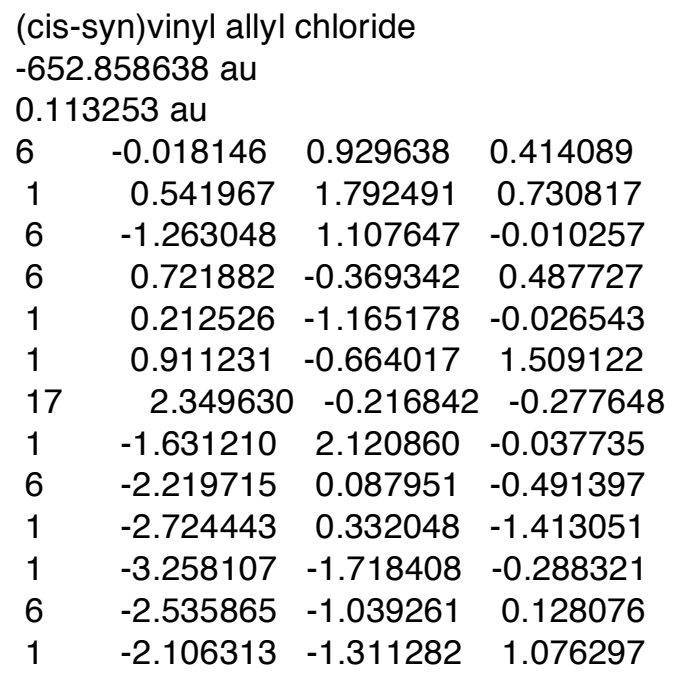

(trans)Me allyl fluoride -254.968768 au

0.1087519 au

$\begin{array}{rrrr}6 & 1.101750 & 0.371627 & -0.160943 \\ 1 & 1.017024 & 1.311402 & -0.686385 \\ 6 & 0.010406 & -0.176523 & 0.346188 \\ 1 & 0.063494 & -1.120143 & 0.866082 \\ 6 & -1.345875 & 0.446300 & 0.263668 \\ 1 & -1.332082 & 1.363235 & -0.309102 \\ 1 & -1.756582 & 0.642438 & 1.245734 \\ 9 & -2.226526 & -0.417403 & -0.358367 \\ 6 & 2.485044 & -0.206762 & -0.070980 \\ 1 & 2.896753 & -0.369627 & -1.063040 \\ 1 & 2.486953 & -1.152899 & 0.458108 \\ 1 & 3.155223 & 0.474377 & 0.446317\end{array}$

(cis)Me allyl fluoride

-254.965454 au

0.108967 au

$\begin{array}{lrrr}6 & -1.234831 & 0.638646 & -0.073542 \\ 6 & 0.065786 & 0.771047 & 0.139925 \\ 1 & 0.494591 & 1.759461 & 0.115881 \\ 6 & 1.051124 & -0.315424 & 0.438085 \\ 1 & 0.646125 & -1.306948 & 0.311038 \\ 1 & 1.447352 & -0.219320 & 1.441097 \\ 9 & 2.128712 & -0.202664 & -0.418542 \\ 1 & -1.793323 & 1.543503 & -0.254120 \\ 6 & -2.061041 & -0.618486 & -0.094336 \\ 1 & -2.614502 & -0.684316 & -1.026517 \\ 1 & -2.792233 & -0.602453 & 0.709465 \\ 1 & -1.472647 & -1.520648 & 0.009234\end{array}$




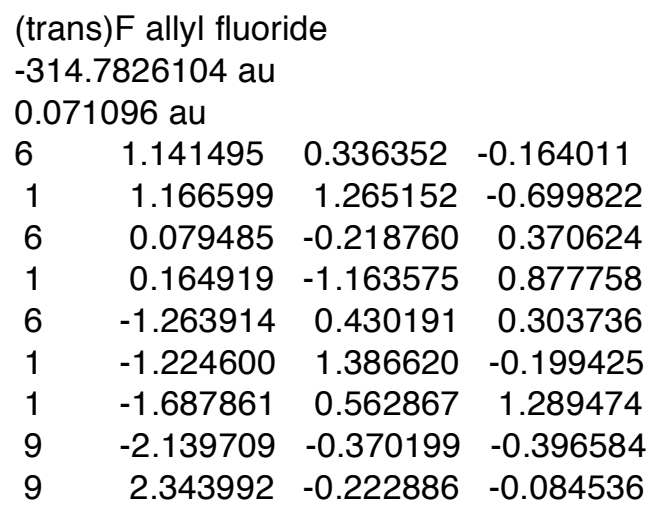

(cis)F allyl fluoride

-314.782910 au

0.071267 au

$\begin{array}{lrrr}6 & -1.313941 & 0.529476 & -0.078479 \\ 6 & -0.040941 & 0.747614 & 0.155059 \\ 1 & 0.294905 & 1.768770 & 0.160354 \\ 6 & 0.979893 & -0.312598 & 0.422420 \\ 1 & 0.597223 & -1.302270 & 0.228721 \\ 1 & 1.346017 & -0.257385 & 1.438970 \\ 9 & 2.066138 & -0.112106 & -0.401362 \\ 1 & -2.052729 & 1.283074 & -0.261219 \\ 9 & -1.836747 & -0.696687 & -0.105397\end{array}$

(trans) Cl allyl fluoride

-674.828226 au

0.069477 au

$\begin{array}{lrrr}6 & 0.605290 & 0.465240 & -0.155555 \\ 1 & 0.552413 & 1.398069 & -0.682001 \\ 6 & -0.442885 & -0.147793 & 0.350182 \\ 1 & -0.338939 & -1.090231 & 0.858586 \\ 6 & -1.820461 & 0.430992 & 0.264326 \\ 1 & -1.837472 & 1.350913 & -0.302813 \\ 1 & -2.232355 & 0.607117 & 1.249012 \\ 9 & -2.658721 & -0.462767 & -0.361839 \\ 17 & 2.219599 & -0.152447 & -0.036467\end{array}$

(cis) Cl allyl fluoride

-674.8279529 au

0.069448 au

$\begin{array}{lrrr}6 & -0.775013 & 0.926926 & -0.024664 \\ 6 & 0.529678 & 0.823101 & 0.107750 \\ 1 & 1.102004 & 1.734673 & 0.117605 \\ 6 & 1.319033 & -0.440137 & 0.271425 \\ 1 & 0.865764 & -1.277135 & -0.236557 \\ 1 & 1.454640 & -0.684357 & 1.317246 \\ 9 & 2.570872 & -0.249720 & -0.264945 \\ 1 & -1.278800 & 1.866666 & -0.121941 \\ 17 & -1.866097 & -0.426571 & -0.048171\end{array}$

(trans) $\mathrm{CN}$ allyl fluoride

Allyl SN2 SI v2a.doc 


\begin{tabular}{llrr}
\multicolumn{4}{l}{$-307.664558 \mathrm{au}$} \\
$0.078077 \mathrm{au}$ \\
6 & 0.726881 & 0.542975 & -0.150987 \\
6 & -0.324977 & -0.135370 & 0.277858 \\
1 & -0.209333 & -1.121068 & 0.694224 \\
6 & -1.718464 & 0.410703 & 0.245456 \\
1 & -1.785137 & 1.314696 & -0.342906 \\
1 & -2.077668 & 0.603360 & 1.248012 \\
9 & -2.555301 & -0.522787 & -0.312313 \\
1 & 0.637558 & 1.522746 & -0.584399 \\
6 & 2.067875 & 0.018538 & -0.065689 \\
7 & 3.132057 & -0.376534 & -0.006277
\end{tabular}

(cis)CN allyl fluoride

-307.664408 au

0.078189 au

$\begin{array}{lrrr}6 & -0.826204 & 1.029465 & -0.051928 \\ 6 & 0.477339 & 0.862161 & 0.110351 \\ 1 & 1.114106 & 1.729352 & 0.101482 \\ 6 & 1.176847 & -0.443821 & 0.338628 \\ 1 & 0.611174 & -1.286822 & -0.028240 \\ 1 & 1.390314 & -0.582065 & 1.390918 \\ 9 & 2.379523 & -0.416370 & -0.321929 \\ 1 & -1.250113 & 2.007250 & -0.181906 \\ 6 & -1.768751 & -0.063885 & -0.057756 \\ 7 & -2.519510 & -0.917701 & -0.060093\end{array}$

(trans) $\mathrm{OH}$ allyl fluoride

-290.785534 au

0.084007 au

$\begin{array}{rrrr}6 & 0.053062 & -0.260480 & 0.381957 \\ 1 & 0.131718 & -1.233958 & 0.834114 \\ 6 & 1.127346 & 0.318874 & -0.119257 \\ 6 & -1.285437 & 0.394212 & 0.347464 \\ 1 & -1.231513 & 1.398995 & -0.050269 \\ 1 & -1.743402 & 0.424682 & 1.326751 \\ 9 & -2.154605 & -0.316122 & -0.460221 \\ 1 & 1.083520 & 1.286528 & -0.590854 \\ 8 & 2.348523 & -0.251429 & -0.064208 \\ 1 & 2.993108 & 0.264644 & -0.525076\end{array}$

(cis) $\mathrm{OH}$ allyl fluoride

-290.789459 au

0.085048 au

$\begin{array}{rrrr}6 & -0.025466 & 1.073127 & -0.000437 \\ 1 & -0.000159 & 2.148875 & -0.001578 \\ 6 & -1.219639 & 0.499788 & -0.000394 \\ 6 & 1.319065 & 0.413105 & 0.001198 \\ 1 & 1.890592 & 0.670985 & 0.882998 \\ 1 & 1.893753 & 0.673935 & -0.877650 \\ 9 & 1.201261 & -0.969670 & -0.001235 \\ 1 & -2.108093 & 1.101587 & -0.001498 \\ 8 & -1.524172 & -0.805860 & 0.000744\end{array}$

Allyl SN2 SI v2a.doc

- S33- 


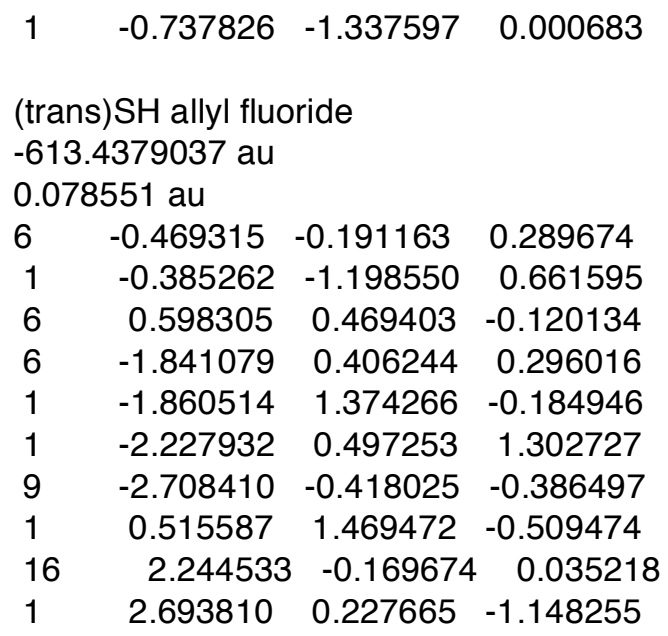




$\begin{array}{lrrr}6 & 1.819469 & -0.402557 & 0.202762 \\ 1 & 1.953368 & -1.223660 & -0.487476 \\ 1 & 2.285384 & -0.647140 & 1.148584 \\ 9 & 2.510072 & 0.681308 & -0.301994 \\ 1 & -0.379193 & -1.580579 & -0.804228 \\ 6 & -2.051857 & -0.420332 & -0.040376 \\ 1 & -2.703508 & -1.275414 & 0.044428 \\ 6 & -2.580617 & 0.794025 & -0.033773 \\ 1 & -3.639127 & 0.938978 & 0.086935 \\ 1 & -1.979865 & 1.677197 & -0.162358\end{array}$

\begin{tabular}{|c|c|c|c|}
\hline & $\begin{array}{l}5755 \mathrm{au} \\
12 \mathrm{au}\end{array}$ & & \\
\hline 6 & 0.618689 & 0.793235 & 0.143348 \\
\hline & 1.128970 & 1.740731 & 0.100079 \\
\hline 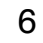 & -0.700749 & 0.772932 & -0.008933 \\
\hline 6 & 1.519037 & -0.374881 & 0.400898 \\
\hline 1 & 1.045869 & -1.328226 & 0.225033 \\
\hline 1 & 1.908148 & -0.352073 & 1.411148 \\
\hline 1 & -1.198638 & 1.716098 & -0.164108 \\
\hline & 2.609352 & -0.299778 & -0.440713 \\
\hline 6 & -1.585048 & -0.400378 & 0.012485 \\
\hline 1 & -1.138462 & -1.371673 & 0.133681 \\
\hline 6 & -2.902509 & -0.315393 & -0.114775 \\
\hline 1 & -3.525608 & -1.191053 & -0.101089 \\
\hline & -3.400964 & 0.631110 & -0.236465 \\
\hline
\end{tabular}

(cis-syn)vinyl allyl fluoride
-292.812053 au
$0.114879 \mathrm{au}$
$\begin{array}{lrrr}6 & 0.529280 & 0.830335 & 0.293334 \\ 1 & 1.192992 & 1.624957 & 0.590204 \\ 6 & -0.744667 & 1.118518 & 0.058580 \\ 6 & 1.191421 & -0.507419 & 0.175315 \\ 1 & 0.662359 & -1.174903 & -0.487643 \\ 1 & 1.315651 & -0.982418 & 1.140593 \\ 1 & -1.044412 & 2.149907 & 0.151849 \\ 6 & -1.819539 & 0.193156 & -0.354274 \\ 1 & -2.475377 & 0.566760 & -1.124882 \\ 1 & -2.874823 & -1.608882 & -0.200455 \\ 6 & -2.062147 & -1.003877 & 0.159670 \\ 1 & -1.480912 & -1.409676 & 0.968987 \\ 9 & 2.459825 & -0.327780 & -0.337155\end{array}$

Table S2. Energy barriers $\left(\mathrm{Kcal} \mathrm{mol}^{-1}\right)$ of identity ion-pair $\mathrm{S}_{\mathrm{N}} 2$ reactions of MX with allyl X. The substituent $(\mathrm{Y})$ on the central $(\beta)$ and terminal $(\gamma)$ allylic carbon atoms and the leaving and attacking nucleophile $(\mathrm{X})$ are given. The reaction angle $(\mathrm{X}-\mathrm{C}-\mathrm{X})$ and some important bond lengths are also given. 


\begin{tabular}{|c|c|c|c|c|c|c|c|c|}
\hline \multirow[t]{2}{*}{$\mathrm{Y}$} & \multirow[t]{2}{*}{$\mathrm{M}$} & \multirow[t]{2}{*}{$X$} & \multirow[t]{2}{*}{$\Delta \mathrm{E}^{*}$} & \multirow{2}{*}{$\begin{array}{c}\text { Reaction } \\
\text { angle } \\
\text { deg }\end{array}$} & \multicolumn{4}{|c|}{ Bond lengths, $\AA^{\mathrm{a}}$} \\
\hline & & & & & $\mathrm{M}-\mathrm{X}$ & C-X & $\mathrm{C}_{\alpha}-\mathrm{C}_{\beta}$ & $\mathrm{C}_{\beta}-\mathrm{C}_{\gamma}$ \\
\hline $\mathrm{H}$ & $\mathrm{Li}$ & $\mathrm{F}$ & 28.54 & 73.25 & 1.692 & 2.489 & 1.414 & 1.344 \\
\hline $\mathrm{H}$ & $\mathrm{Li}$ & $\mathrm{Cl}$ & 12.49 & 82.75 & 2.214 & 2.988 & 1.407 & 1.347 \\
\hline $\mathrm{H}$ & $\mathrm{Na}$ & $\mathrm{F}$ & 33.18 & 87.49 & 2.070 & 2.369 & 1.424 & 1.339 \\
\hline$\beta-F$ & $\mathrm{Li}$ & $\mathrm{F}$ & 34.78 & 74.10 & 1.697 & 2.439 & 1.411 & 1.334 \\
\hline$\beta-\mathrm{Me}$ & $\mathrm{Li}$ & $\mathrm{F}$ & 28.32 & 73.08 & 1.692 & 2.500 & 1.419 & 1.346 \\
\hline$\beta-F$ & $\mathrm{Li}$ & $\mathrm{Cl}$ & 19.65 & 84.1 & 2.222 & 2.924 & 1.406 & 1.337 \\
\hline$\beta-\mathrm{Me}$ & $\mathrm{Li}$ & $\mathrm{Cl}$ & 11.64 & 82.48 & 2.213 & 3.002 & 1.412 & 1.349 \\
\hline$\beta-F$ & $\mathrm{Na}$ & $\mathrm{F}$ & 38.28 & 89.40 & 2.081 & 2.304 & 1.423 & 1.328 \\
\hline$\beta-\mathrm{Me}$ & $\mathrm{Na}$ & $\mathrm{F}$ & 33.04 & 87.38 & 2.069 & 2.379 & 1.429 & 1.340 \\
\hline \multicolumn{9}{|l|}{ Trans } \\
\hline$\gamma-F$ & $\mathrm{Li}$ & $\mathrm{F}$ & 25.78 & 72.36 & 1.691 & 2.509 & 1.396 & 1.347 \\
\hline$\gamma-\mathrm{Cl}$ & $\mathrm{Li}$ & $\mathrm{F}$ & 28.74 & 72.77 & 1.692 & 2.498 & 1.404 & 1.348 \\
\hline$\gamma-\mathrm{CN}$ & $\mathrm{Li}$ & $\mathrm{F}$ & 37.67 & 74.50 & 1.699 & 2.427 & 1.425 & 1.341 \\
\hline$\gamma$-Me & $\mathrm{Li}$ & $\mathrm{F}$ & 22.44 & 71.91 & 1.687 & 2.546 & 1.398 & 1.356 \\
\hline$\gamma-\mathrm{SH}$ & $\mathrm{Li}$ & $\mathrm{F}$ & 21.75 & 70.86 & 1.685 & 2.583 & 1.387 & 1.367 \\
\hline$\gamma-\mathrm{OH}$ & $\mathrm{Li}$ & $\mathrm{F}$ & 28.65 & 70.86 & 1.685 & 2.583 & 1.387 & 1.367 \\
\hline$\gamma$-vinyl(a) & $\mathrm{Li}$ & $\mathrm{F}$ & 21.35 & 71.01 & 1.684 & 2.588 & 1.390 & 1.366 \\
\hline$\gamma$-vinyl(s) & $\mathrm{Li}$ & $\mathrm{F}$ & 21.75 & 71.14 & 1.684 & 2.583 & 1.389 & 1.367 \\
\hline$\gamma-F$ & $\mathrm{Li}$ & $\mathrm{Cl}$ & 9.62 & 81.56 & 2.213 & 3.019 & 1.390 & 1.352 \\
\hline$\gamma-\mathrm{Cl}$ & $\mathrm{Li}$ & $\mathrm{Cl}$ & 12.62 & 81.98 & 2.212 & 3.008 & 1.397 & 1.353 \\
\hline$\gamma-\mathrm{CN}$ & $\mathrm{Li}$ & $\mathrm{Cl}$ & 22.37 & 84.94 & 2.228 & 2.900 & 1.420 & 1.343 \\
\hline$\gamma$-Me & $\mathrm{Li}$ & $\mathrm{Cl}$ & 5.59 & 80.65 & 2.206 & 3.072 & 1.390 & 1.361 \\
\hline$\gamma-\mathrm{OH}$ & $\mathrm{Li}$ & $\mathrm{Cl}$ & 12.24 & 79.78 & 2.200 & 3.125 & 1.374 & 1.378 \\
\hline$\gamma$-vinyl(a) & $\mathrm{Li}$ & $\mathrm{Cl}$ & 3.76 & 78.76 & 2.199 & 3.153 & 1.379 & 1.374 \\
\hline$\gamma$-vinyl(s) & $\mathrm{Li}$ & $\mathrm{Cl}$ & 4.39 & 79.40 & 2.200 & 3.131 & 1.379 & 1.374 \\
\hline$\gamma-F$ & $\mathrm{Na}$ & $\mathrm{F}$ & 30.53 & 86.09 & 2.067 & 2.390 & 1.407 & 1.340 \\
\hline$\gamma-\mathrm{Cl}$ & $\mathrm{Na}$ & $\mathrm{F}$ & 33.35 & 86.87 & 2.070 & 2.374 & 1.416 & 1.340 \\
\hline$\gamma-\mathrm{CN}$ & $\mathrm{Na}$ & $\mathrm{F}$ & 40.57 & 90.73 & 2.090 & 2.288 & 1.438 & 1.335 \\
\hline$\gamma$-Me & $\mathrm{Na}$ & $\mathrm{F}$ & 28.08 & 85.39 & 2.060 & 2.431 & 1.408 & 1.349 \\
\hline$\gamma-\mathrm{SH}$ & $\mathrm{Na}$ & $\mathrm{F}$ & 27.79 & 84.11 & 2.056 & 2.461 & 1.397 & 1.358 \\
\hline$\gamma-\mathrm{OH}$ & $\mathrm{Na}$ & $\mathrm{F}$ & 21.26 & 82.03 & 2.050 & 2.521 & 1.382 & 1.364 \\
\hline$\gamma$-vinyl(a) & $\mathrm{Na}$ & $\mathrm{F}$ & 27.60 & 84.30 & 2.055 & 2.465 & 1.400 & 1.358 \\
\hline$\gamma$-vinyl(s) & $\mathrm{Na}$ & $\mathrm{F}$ & 27.97 & 84.49 & 2.056 & 2.460 & 1.401 & 1.358 \\
\hline \multicolumn{9}{|l|}{ Cis } \\
\hline$\gamma-\mathrm{F}$ & $\mathrm{Li}$ & $\mathrm{F}$ & 29.41 & 73.36 & 1.693 & 2.485 & 1.404 & 1.346 \\
\hline$\gamma-\mathrm{Cl}$ & $\mathrm{Li}$ & $\mathrm{F}$ & 32.30 & 73.38 & 1.693 & 2.486 & 1.409 & 1.349 \\
\hline$\gamma-\mathrm{CN}$ & $\mathrm{Li}$ & $\mathrm{F}$ & 40.51 & 75.30 & 1.702 & 2.407 & 1.430 & 1.341 \\
\hline$\gamma$-Me & $\mathrm{Li}$ & $\mathrm{F}$ & 22.74 & 71.24 & 1.687 & 2.562 & 1.397 & 1.360 \\
\hline$\gamma$-SH & $\mathrm{Li}$ & $\mathrm{F}$ & 24.08 & 71.26 & 1.685 & 2.570 & 1.388 & 1.370 \\
\hline$\gamma-\mathrm{OH}$ & $\mathrm{Li}$ & $\mathrm{F}$ & 21.34 & 71.35 & 1.684 & 2.587 & 1.383 & 1.370 \\
\hline \multicolumn{4}{|c|}{ Allyl SN2 SI v2a.doc } & \multicolumn{2}{|c|}{ - S36- } & & & \\
\hline
\end{tabular}




\begin{tabular}{ccccccccc}
$\gamma$-vinyl(a) & $\mathrm{Li}$ & $\mathrm{F}$ & 21.85 & 71.01 & 1.684 & 2.588 & 1.400 & 1.363 \\
$\gamma$-vinyl(s) & $\mathrm{Li}$ & $\mathrm{F}$ & 68.69 & 71.14 & 1.684 & 2.583 & 1.531 & 1.332 \\
$\gamma-\mathrm{F}$ & $\mathrm{Li}$ & $\mathrm{Cl}$ & 13.21 & 82.89 & 2.215 & 2.985 & 1.398 & 1.350 \\
$\gamma-\mathrm{Cl}$ & $\mathrm{Li}$ & $\mathrm{Cl}$ & 15.68 & 82.96 & 2.215 & 2.984 & 1.401 & 1.354 \\
$\gamma-\mathrm{CN}$ & $\mathrm{Li}$ & $\mathrm{Cl}$ & 24.84 & 86.07 & 2.232 & 2.875 & 1.425 & 1.343 \\
$\gamma-\mathrm{Me}$ & $\mathrm{Li}$ & $\mathrm{Cl}$ & 5.89 & 79.70 & 2.205 & 3.098 & 1.389 & 1.366 \\
$\gamma-\mathrm{SH}$ & $\mathrm{Li}$ & $\mathrm{Cl}$ & 6.76 & 79.62 & 2.202 & 3.115 & 1.377 & 1.379 \\
$\gamma-\mathrm{OH}$ & $\mathrm{Li}$ & $\mathrm{Cl}$ & 1.61 & 79.78 & 2.200 & 3.125 & 1.374 & 1.377 \\
$\gamma-\mathrm{F}$ & $\mathrm{Na}$ & $\mathrm{F}$ & 33.86 & 87.82 & 2.072 & 2.363 & 1.416 & 1.339 \\
$\gamma-\mathrm{Cl}$ & $\mathrm{Na}$ & $\mathrm{F}$ & 36.70 & 88.27 & 2.074 & 2.354 & 1.422 & 1.341 \\
$\gamma-\mathrm{CN}$ & $\mathrm{Na}$ & $\mathrm{F}$ & 43.00 & 92.89 & 2.097 & 2.253 & 1.444 & 1.335 \\
$\gamma-\mathrm{Me}$ & $\mathrm{Na}$ & $\mathrm{F}$ & 28.47 & 84.99 & 2.060 & 2.436 & 1.408 & 1.353 \\
$\gamma-\mathrm{SH}$ & $\mathrm{Na}$ & $\mathrm{F}$ & 27.69 & 84.88 & 2.059 & 2.441 & 1.401 & 1.360 \\
$\gamma$-OH & $\mathrm{Na}$ & $\mathrm{F}$ & 29.97 & 84.57 & 2.054 & 2.473 & 1.393 & 1.362 \\
$\gamma$-vinyl(a) & $\mathrm{Na}$ & $\mathrm{F}$ & 28.11 & 83.51 & 2.054 & 2.476 & 1.400 & 1.363 \\
$\gamma$-vinyl(s) & $\mathrm{Na}$ & $\mathrm{F}$ & 73.54 & 84.49 & 2.056 & 2.460 & 1.531 & 1.332 \\
\hline
\end{tabular}

(a) Bond lengths calculated in addition for (a) allyl fluoride $\mathrm{C}-\mathrm{X}=1.379 ; \mathrm{C}_{\alpha}-\mathrm{C}_{\beta}=1.496$; $\mathrm{C}_{\beta}-\mathrm{C}_{\gamma}=1.321$ (b) $\mathrm{Li}-\mathrm{F}=1.575$ (c) $\mathrm{Li}-\mathrm{Cl}=2.066$ (d) $\mathrm{Na}-\mathrm{F}=1.928$

Table S3. Reduced natural charges on allylic carbon centers, attacking and leaving groups, $\mathrm{X}$, and on the substituents, Y, for ion pair SN2 reactions of MX with allyl X.

\begin{tabular}{ccccccccc}
\hline $\mathrm{Y}$ & $\mathrm{M}$ & $\mathrm{X}$ & \multicolumn{7}{c}{ Reduced charges* } \\
\cline { 3 - 8 } & & & \multicolumn{7}{c}{$\mathrm{C}_{\beta}$} & $\mathrm{C}_{\gamma}$ & $\mathrm{M}$ & $\mathrm{X}$ & $\mathrm{Y}$ \\
\cline { 3 - 8 } $\mathrm{H}$ & $\mathrm{Li}$ & $\mathrm{F}$ & 0.768 & -0.207 & 0.376 & 0.962 & -0.950 & $\ldots \ldots$ \\
$\mathrm{H}$ & $\mathrm{Li}$ & $\mathrm{Cl}$ & 0.688 & -0.199 & 0.404 & 0.897 & -0.895 & $\ldots \ldots$ \\
$\mathrm{H}$ & $\mathrm{Na}$ & $\mathrm{F}$ & 0.767 & -0.194 & 0.330 & 0.981 & -0.942 & $\ldots \ldots$ \\
$\beta-\mathrm{F}$ & $\mathrm{Li}$ & $\mathrm{F}$ & 0.766 & 0.217 & 0.322 & 0.963 & -0.944 & -0.380 \\
$\beta-\mathrm{Me}$ & $\mathrm{Li}$ & $\mathrm{F}$ & 0.777 & -0.272 & 0.359 & 0.962 & -0.951 & 0.077 \\
$\beta-\mathrm{F}$ & $\mathrm{Li}$ & $\mathrm{Cl}$ & 0.670 & 0.225 & 0.343 & 0.900 & -0.881 & -0.375 \\
$\beta-\mathrm{Me}$ & $\mathrm{Li}$ & $\mathrm{Cl}$ & 0.697 & -0.271 & 0.388 & 0.897 & -0.897 & 0.084 \\
$\beta-\mathrm{F}$ & $\mathrm{Na}$ & $\mathrm{F}$ & 0.761 & 0.241 & 0.262 & 0.981 & -0.930 & -0.385 \\
$\beta-\mathrm{Me}$ & $\mathrm{Na}$ & $\mathrm{F}$ & 0.779 & -0.257 & 0.312 & 0.980 & -0.943 & 0.070 \\
\hline $\mathrm{Trans}$ & & & & & & & & \\
$\gamma-\mathrm{F}$ & $\mathrm{Li}$ & $\mathrm{F}$ & 0.735 & -0.264 & 0.824 & 0.962 & -0.952 & -0.353 \\
$\gamma-\mathrm{Cl}$ & $\mathrm{Li}$ & $\mathrm{F}$ & 0.754 & -0.196 & 0.295 & 0.962 & -0.951 & 0.088 \\
$\gamma-\mathrm{CN}$ & $\mathrm{Li}$ & $\mathrm{F}$ & 0.797 & -0.106 & 0.228 & 0.963 & -0.942 & 0.231 \\
& & & & & & & & -0.229 \\
$\gamma-\mathrm{Me}$ & $\mathrm{Li}$ & $\mathrm{F}$ & 0.732 & -0.227 & 0.384 & 0.961 & -0.956 & 0.061 \\
$\gamma-\mathrm{SH}$ & $\mathrm{Li}$ & $\mathrm{F}$ & 0.692 & -0.222 & 0.152 & 0.961 & -0.958 & 0.334 \\
$\gamma-\mathrm{OH}$ & $\mathrm{Li}$ & $\mathrm{F}$ & 0.702 & -0.248 & 0.692 & 0.961 & -0.958 & -0.192 \\
$\gamma-\mathrm{vinyl}(\mathrm{a})$ & $\mathrm{Li}$ & $\mathrm{F}$ & 0.696 & -0.214 & 0.344 & 0.961 & -0.959 & -0.098
\end{tabular}




\begin{tabular}{|c|c|c|c|c|c|c|c|c|}
\hline$\gamma$-vinyl(s) & $\mathrm{Li}$ & $\mathrm{F}$ & 0.692 & -0.218 & 0.358 & 0.961 & -0.959 & $\begin{array}{r}0.229 \\
-0.111 \\
0.235\end{array}$ \\
\hline$\gamma-\mathrm{F}$ & $\mathrm{Li}$ & $\mathrm{Cl}$ & 0.658 & -0.252 & 0.845 & 0.897 & -0.902 & -0.345 \\
\hline$\gamma-\mathrm{Cl}$ & $\mathrm{Li}$ & $\mathrm{Cl}$ & 0.671 & -0.191 & 0.315 & 0.897 & -0.899 & 0.105 \\
\hline$\gamma-\mathrm{CN}$ & $\mathrm{Li}$ & $\mathrm{Cl}$ & 0.696 & -0.097 & 0.241 & 0.901 & -0.874 & $\begin{array}{c}0.227 \\
-0.220\end{array}$ \\
\hline$\gamma-\mathrm{Me}$ & $\mathrm{Li}$ & $\mathrm{Cl}$ & 0.659 & -0.219 & 0.413 & 0.895 & -0.908 & 0.069 \\
\hline$\gamma-\mathrm{OH}$ & $\mathrm{Li}$ & $\mathrm{Cl}$ & 0.632 & -0.240 & 0.724 & 0.893 & -0.912 & -0.183 \\
\hline$\gamma$-vinyl(a) & $\mathrm{Li}$ & $\mathrm{Cl}$ & 0.615 & -0.205 & 0.376 & 0.893 & -0.916 & $\begin{array}{c}-0.108 \\
0.262\end{array}$ \\
\hline$\gamma$-vinyl(s) & $\mathrm{Li}$ & $\mathrm{Cl}$ & 0.615 & -0.209 & 0.387 & 0.893 & -0.915 & $\begin{array}{c}-0.118 \\
0.263\end{array}$ \\
\hline$\gamma-\mathrm{F}$ & $\mathrm{Na}$ & $\mathrm{F}$ & 0.745 & -0.260 & 0.787 & 0.981 & -0.946 & -0.361 \\
\hline$\gamma-\mathrm{Cl}$ & $\mathrm{Na}$ & $\mathrm{F}$ & 0.762 & -0.188 & 0.264 & 0.981 & -0.943 & 0.066 \\
\hline$\gamma-\mathrm{CN}$ & $\mathrm{Na}$ & $\mathrm{F}$ & 0.783 & -0.08 & 0.175 & 0.982 & -0.924 & $\begin{array}{c}0.247 \\
-0.257\end{array}$ \\
\hline$\gamma$-Me & $\mathrm{Na}$ & $\mathrm{F}$ & 0.744 & -0.220 & 0.345 & 0.980 & -0.952 & 0.056 \\
\hline$\gamma-\mathrm{SH}$ & $\mathrm{Na}$ & $\mathrm{F}$ & 0.719 & -0.221 & 0.130 & 0.980 & -0.955 & 0.304 \\
\hline$\gamma-\mathrm{OH}$ & $\mathrm{Na}$ & $\mathrm{F}$ & 0.677 & -0.267 & 0.711 & 0.980 & -0.962 & -0.176 \\
\hline$\gamma$-vinyl(a) & $\mathrm{Na}$ & $\mathrm{F}$ & 0.721 & -0.209 & 0.311 & 0.980 & -0.956 & $\begin{array}{c}-0.085 \\
0.194\end{array}$ \\
\hline$\gamma$-vinyl(s) & $\mathrm{Na}$ & $\mathrm{F}$ & 0.721 & -0.211 & 0.321 & 0.980 & -0.956 & $\begin{array}{c}-0.095 \\
0.195 \\
\end{array}$ \\
\hline \multicolumn{9}{|l|}{ Cis } \\
\hline$\gamma-\mathrm{F}$ & $\mathrm{Li}$ & $\mathrm{F}$ & 0.772 & -0.291 & 0.81 & 0.962 & -0.950 & -0.353 \\
\hline$\gamma-\mathrm{Cl}$ & $\mathrm{Li}$ & $\mathrm{F}$ & 0.779 & -0.221 & 0.286 & 0.962 & -0.949 & 0.092 \\
\hline$\gamma-\mathrm{CN}$ & $\mathrm{Li}$ & $\mathrm{F}$ & 0.811 & -0.116 & 0.222 & 0.963 & -0.939 & $\begin{array}{c}0.218 \\
-0.222\end{array}$ \\
\hline$\gamma-\mathrm{Me}$ & $\mathrm{Li}$ & $\mathrm{F}$ & 0.731 & -0.240 & 0.39 & 0.962 & -0.956 & 0.071 \\
\hline$\gamma-\mathrm{SH}$ & $\mathrm{Li}$ & $\mathrm{F}$ & 0.705 & -0.245 & 0.145 & 0.961 & -0.957 & 0.347 \\
\hline$\gamma-\mathrm{OH}$ & $\mathrm{Li}$ & $\mathrm{F}$ & 0.705 & -0.301 & 0.718 & 0.961 & -0.959 & -0.163 \\
\hline$\gamma$-vinyl(a) & $\mathrm{Li}$ & $\mathrm{F}$ & 0.707 & -0.225 & 0.340 & 0.961 & -0.958 & $\begin{array}{r}-0.078 \\
0.211\end{array}$ \\
\hline$\gamma$-vinyl(s) & $\mathrm{Li}$ & $\mathrm{F}$ & 0.801 & -0.208 & 0.257 & 0.962 & -0.955 & $\begin{array}{r}-0.143 \\
0.242\end{array}$ \\
\hline$\gamma-F$ & $\mathrm{Li}$ & $\mathrm{Cl}$ & 0.687 & -0.278 & 0.831 & 0.897 & -0.895 & -0.348 \\
\hline$\gamma-\mathrm{Cl}$ & $\mathrm{Li}$ & $\mathrm{Cl}$ & 0.689 & -0.213 & 0.306 & 0.897 & -0.893 & 0.106 \\
\hline$\gamma-\mathrm{CN}$ & $\mathrm{Li}$ & $\mathrm{Cl}$ & 0.703 & -0.102 & 0.235 & 0.902 & -0.866 & $\begin{array}{c}0.213 \\
-0.214\end{array}$ \\
\hline$\gamma-\mathrm{Me}$ & $\mathrm{Li}$ & $\mathrm{Cl}$ & 0.655 & -0.232 & 0.424 & 0.910 & -0.895 & 0.078 \\
\hline$\gamma-\mathrm{SH}$ & $\mathrm{Li}$ & $\mathrm{Cl}$ & 0.617 & -0.231 & 0.162 & 0.894 & -0.912 & 0.383 \\
\hline$\gamma-\mathrm{OH}$ & $\mathrm{Li}$ & $\mathrm{Cl}$ & 0.630 & -0.285 & 0.744 & 0.893 & -0.915 & -0.151 \\
\hline$\gamma-\mathrm{F}$ & $\mathrm{Na}$ & $\mathrm{F}$ & 0.773 & -0.280 & 0.770 & 0.981 & -0.941 & -0.362 \\
\hline$\gamma-\mathrm{Cl}$ & $\mathrm{Na}$ & $\mathrm{F}$ & 0.781 & -0.204 & 0.252 & 0.981 & -0.938 & 0.067 \\
\hline
\end{tabular}




\begin{tabular}{ccccccccc}
$\gamma$-CN & $\mathrm{Na}$ & $\mathrm{F}$ & 0.786 & -0.082 & 0.164 & 0.982 & -0.916 & 0.239 \\
& & & & & & & & -0.256 \\
$\gamma-\mathrm{Me}$ & $\mathrm{Na}$ & $\mathrm{F}$ & 0.748 & -0.231 & 0.348 & 0.980 & -0.952 & 0.061 \\
$\gamma-\mathrm{SH}$ & $\mathrm{Na}$ & $\mathrm{F}$ & 0.733 & -0.239 & 0.122 & 0.980 & -0.952 & 0.310 \\
$\gamma$-OH & $\mathrm{Na}$ & $\mathrm{F}$ & 0.726 & -0.302 & 0.687 & 0.980 & -0.957 & -0.178 \\
$\gamma$-vinyl(a) & $\mathrm{Na}$ & $\mathrm{F}$ & 0.722 & -0.221 & 0.316 & 0.980 & -0.957 & -0.068 \\
& & & & & & & & 0.186 \\
$\gamma$-vinyl(s) & $\mathrm{Na}$ & $\mathrm{F}$ & 0.806 & -0.192 & 0.228 & 0.980 & -0.950 & -0.129 \\
& & & & & & & & 0.206 \\
\hline
\end{tabular}

* Reduced charges calculated in addition for (a) allyl fluoride: $\mathrm{C}_{\alpha}=0.451 ; \mathrm{C}_{\beta}=-0.033 \mathrm{C}_{\gamma}$ $=0.027 ; \mathrm{X}=-0.445$ (b) LiF: $\mathrm{Li}=0.979 ; \mathrm{F}=-0.979$ (c) $\mathrm{LiCl}: \mathrm{Li}=0.941 ; \mathrm{Cl}=-0.941$ (d) $\mathrm{NaF}: \mathrm{Na}=0.988, \mathrm{~F}=-0.988$ 